Aus dem chemischen Laboratorium des Institutes für experimentelle Medicin und dem Krankenhaus des Kaisers Alexander III. in Udjelnaja.

\title{
Ueber die 0xydationsprocesse im Organismus der Geisteskranken und die Giftigkeit des Harnes derselben.
}

Von

\author{
Dr. A. J. Juschtschenko.
}

Die Erfolge der mikroskopischen Anatomie, die, im Zusammenhang
mit den Erfolgen der mikroskopischen Technik, so ungeheure Um-
wälzungen auf vielen Gebieten der Medicin bewirkt und gänzlich neue,
rasch sich entwickelnde Zweige der medicinischen Wissenschaften ber-
vorgerufen haben, sind jedoch nur in geringstem Maasse von Einfluss
auf das noch so dunkle Gebiet - der Lehre von den Geisteskrank-
beiten - geblieben. Obne zu übertreiben können wir sagen, dass
unsere jetzigen Kenntnisse von dem Wesen einiger Geisteskrankheiten
sich nur wenig von den zum Beginn des vorigen Jahrhunderts herrsehen-
den Anschauungen unterscheiden. Die mikroskopische Untersuchung
der Organe und besonders des Nervensystems giebt bei gewissen geistigen
Erkrankungen entweder negative Resultate, oder weist nur Verände-
rungen nach, die auch bei anderen Krankheiten, die nicht von Geistes-
störungen begleitet sind, beobachtet werden kömen.
Einige von den mikroskopischen Untersuchungen und in noch
höherem Grade die klinischen Beobachtungen lassen die Frage von der
Entstehung vieler Geisteskrankheiten auf dem Wege der Autointoxi-
cation immer mehr und mehr in den Vordergrund treten. Die klinischen
Beobachtungen veranlassen sogar einige Forscher (Kr äpelin) sich
direct für die Entstehung ganzer Gruppen von Geisteskrankheiten durch
Autointoxication auszusprechen. Unter dem Einfluss dieser Lehre von 
der Autointoxication wurden in der klinischen Psychiatrie einige Methoden von mehr activen Eingriffen, wie die subcutane Injection von physiologischer Kochsalzlösung, physiologische Klysmen zu demselben Zweck usw. ausgearbeitet. Ungeachtet dessen beschäftigt man sich indessen in den speciellen Laboratorien mehr mit Mikroskopie und Psychophysik, als mit chemischen Untersuchungen. Der Grund hiervon liegt meiner Meinung nach in dem fast vollständigen Fehlen bequemer Untersuchungsmethoden, die gestatten könnten, in das Wesen der chemischbiologischen Prozesse und der Störungen in Stoffwechsel bei geistigen Erkrankungen einzudringen. Zur Untersuchung dieser Processe können dem Forscher nur der Harn und einige Tropfen Blut, höchstens 5 bis $10 \mathrm{ccm}$, zur Verfügung stehen. Letzteres kann auch nur bei einigen wenigen Kranken, ohne augenscheinlichen Nachtheil für ihren psychischen Zustand entnommen werden. Solche Blutuntersuchungen, die zum grossten Theil in Italien hauptsächlich an Epileptikern ausgeführt. worden sind, bestätigen die klinischen Beobachtungen von der Autointoxication. Der Untersuchung am meisten zugänglich ist der Harn der Geisteskranken, und in dieser Beziehung ist bereits eine beträchtliche Zahl von Beobachtungen gemacht worden; leider sind aber erst in der letzten Zeit bequemere und genauere Methoden auf dem Gebiete der Harnuntersuchungen ausgearbeitet worden. Ferner entspricht eine erniedrigte oder erböhte Toxicität des Harnes bei weitem nicht immer einer Erhöhung oder Frniedrigung des Toxingehaltes des Blutes bzw. des Körpers. Gerade im Gegentheil ist zuweilen der Harn eines Kranken aus dem Grunde weniger giftig, weil die toxischen Stoffe im Blut zurückgehalten werden. Es ist sogar sehr wohl möglich, dass diejenigen toxischen Substanzen, die sich bei einigen Erkrankungen in erhöhter Menge im Harn vorfinden, gar nicht die nächste Ursache der Autointoxication sind, sondern dass die Hauptfactoren der Krankheit diejenigen Gifte bilden, die aus irgendwelchen Gründen nicht in den Harn übergehen und im Organismus zurückbleiben. Es existiren aber einige Methoden, die uns gestatten, wenngleich auf indirectem und vielleicht einseitigem Wege, Schlüsse über die im Organismus sich abspielenden Vorgänge zu ziehen. $\mathrm{Zu}$ der Zahl dieser gehört auch die Methode von Nencki und Sieber zur Bestimmung der Oxydationsprocesse im lebenden Organismus. Wit der Untersuchang dieser Vorgänge im Organismus der Geisteskranken bin ich seit dem Jahre 1903 im chemischen Laboratorium des Instituts für experimentelle Medicin beschäftigt1).

1) Ich benutze an dieser Stelle die Gelegenheit Frau Dr. N. O. SieberSchumow, unter deren Leitung sich das chemische Laboratorium befindet, 
In. Zusammenhang mit den Oxydationsprocessen untersuchte ich die ganze Methodik und stellte einige Betrachtungen über die Giftigkeit des Harns bei Geisteskranken an; ferner versuchte ich die Veränderungen der Oxydationsprocesse und die Giftigkeit des Harns, die ich bei Geisteskranken beobachtet hatte, experimentell an Kaninchen durch Vergiftung mit Thyreoidin, Spermin und Adrenalin oder durch Entziehung dieser Katatlysatoren, durch Entfernung der entsprechenden Organe, hervorzurufon. An den so behandelten Thieren wurden ausser der Giftigkeit des Harns und den Störungen der Oxydationsvorgänge auch die Veränderungen des Gaswechsels und des Athmungskoefficienten untersucht, so dass auf diese Weise die Reihe der Versuche, die an Kranken nicht ausgefübrt werden konnten, an Thieren erweitert wurde. In der vorliegenden Arbeit muss ich mich aber auf rein klinische Beobachtungen beschränken.

Zur Dntersuchung der Oxydationsprocesse im Organismus benutzte ich die Methode, die bereits im Jahre 1883 von Nencki nnd Sieber 1 ) vorgeschlagen worden war. Diese Methode beruht darauf, dass Benzol $\mathrm{C}_{6} \mathrm{H}_{6}$ sich bei der Einwirkung von molecularem Sauerstoff $-\mathrm{O}_{2}-$, wie z. B. bei Anwesenheit von Wasserstoffsuperoxyd und in ähnlichen Fällen, wenn sich freie Siuerstoffatome in statn nascendi bilden, $z u$ Phenol $\mathrm{C}_{6} \mathrm{H}_{5} \cdot \mathrm{OH}$ oxydirt wird. In den Geweben verläuft nach der Meinung von Nencki und Sieber der Oxydationsprocess dermaassen, dass der mit dem Blute zuströmende moleculare Sauerstoff, erstens bei der Oxydation der labilen, stark reducirenden Eiweissmolecüle des Zellprotoplasmas, und zweitens in den Zellen während ihrer Lebensthätigkeit, bei der Oxydation anderer Verbindungen, welche fähig sind, sich bei Körpertemperatur auf Kosten des molecularen Sauerstoffs zu oxydiren, in freie Sauerstoffatome gespalten wird. Durch diesen activen Sauerstoff wird das Benzol zu Phenol oxydirt. Vielleicht bildet die Menge des oxydirten Benzols eigentlich nur das Maass der Oxydation durch

für den regen Antheil, den sie an meinen Arbeiten genommen, und für die Anleitung und Hülfe meinen herzlichen Dank auszusprechen. Zu grossem Dank sehe ich mich auch allen Collegen im Laboratorium und in der Irrenanstalt des Kaisers Alexander des IIl. verpflichtet, die mich bei meinen Untersuchungen so theilnehmend unterstützt hatten und mir ihre eigenen Beobachtungen mittheilten. Fbenso danke ioh dem Aufseher-Personal der Anstalt, das meine klinischen Beobachtungen durch sorgfältiges Sammeln des Harns bei schwer Geisteskranken wesentlich unterstützt batte.

1) M. Nencki und N. Sieber, Ueber eine nene Methode die physiologische Oxydation zu messen und über den Einfluss der Gifte und Krankheiten auf dieselbe. Pflüger's Archiv. XXXI. S. 319. 
freie Sanerstoffionen im Zellprotoplasma, nicht aber ein Maass der gesammten Oxydationsprocesse im Organismus, wo auch andere Oxydationen ohne die Einwirkung von activem Sauerstoff, verlaufen; so z. B. wird neutrales harnsaures Ammon in einer Luftatmosphäre bei Bruttemperatur leicht zu Uroxansïure oxydirt, und zudem darf nie aus dem Auge gelassen werden, dass ein Unterschied zwischen den Reactionen im Reagensglase und in der lebenden Zelle besteht. Man braucht sich nur der neueren Untersuchungen über die Katalysatoren, über die Bildung von Superoxyden und Peroxyden zu erimern.

Ich beabsichtige an dieser Stelle nicht auf eine kritische Beurtheilung des Werthes oder der Mängel der Methode von Nencki und Sieber einzugehen, vom klinischen Standpunkte aus verdient sie jedenfalls ihrer Einfachheit, bequemen Anwendbarkeit und Unschädlichkeit wegen das allergrösste Interesse.

Das in den Organismus eingeführte Benzol wird theils unoxydirt durch die Lungen und den Darmkanal ansgeschieden, theils unter dem Einfluss der Lebenstbätigkeit des Protoplasmas zu Phenol $\mathrm{C}_{6} \mathrm{H}_{5} \cdot \mathrm{OH}$, und gleichfalls zu Brenzkatechin und Hydrochinon $\mathrm{C}_{6} \mathrm{H}_{4}(\mathrm{OH})_{2}$ oxydirt. Das Brenzkatechin und Hydrochinon findet sich im Harn nur in unbedeutender Menge und unterlag nicht der Bestimmung. Das Phenol hingegen wird im Harn nicht im freien Zustande, sondern an Schwefelsäure gekuppelt, als eine Aetherschwefelsäure $\mathrm{SO}_{2}-\mathrm{OH}-\mathrm{O}-\mathrm{C}_{6} \mathrm{H}_{5}$ ausgeschieden.

Nach dieser Methode wurden von den Autoren interessante Beobachtungen über die Oxydationsprocesse bei Gesunden, unter dem Einfluss von Hunger, nach Vergiftung mit Phosphor, Arsen, Chloroform und Aether, und ebenso an Kranken, die an verschiedenen Formen von Blutarmuth, Lungenentzündung und Pseudohypertrophie der Muskeln litten, vorgenommen. Nach der gleichen Methode arbeiteten auch mehrere andere Forscber und ich weise hier auf die mir bekannten Arbeiten von Brzesinski ${ }^{1}$, Simanowsky und Schumoff ${ }^{2}$ ) Belogolow off ${ }^{3}$ ) und Freund $d^{4}$ ) hin.

1) Brzesinslrj, Beiträge zur Kenntniss der Oxydation im Organismus bei Krankheiten und Vergiftungen. Bern. 1883.

2) Simanowsky und Schumoff, Ueber den Einfluss des Alkohols und des Morphiums auf die physiologische Oxydation. Bonn. 1884.

3) Belogolow off, Zur Frage äber den Einfluss der erschwerten Athmung durch die Nase auf die Morphologie des Blutes und die Oxydationsprocesse. Dissert. S. Petersburg. 1903. (Russisch.)

4) Freund, Zur Kenntniss der Oxydationsvorgänge bei gesunden und kranken Säuglingen. Verhandlungen ... der Gesellschaft für Kinderheilkunde. Wiesbaden. 1902. 
Durch die Beobachtungen von Nencki und Sieber wurde festgestellt, dass Gesunde gewöhnlich ungefähr ein Drittel des in den Organismus eingeführten Benzols zu Phenol oxydiren; zudem scheidet eine und dieselbe Versuchsperson bei wiederholten Untersuchungen nach Einführung einer und derselben Benzolmenge und bei unverändertem Zustand des Organismus fast die gleichen Phenolmengen aus; hierbei ist es ganz einerlei ob das Benzol subcutan oder per os eingeführt wird, ob die Person hungert oder die versehiedenartigsten Speisen geniesst, ob sie viel Flüssigkeit zu sich nimmt oder dürstet. Nach Durchführung einer grossen Zahl von Versuchen mit Oxydation von Benzol an Menschen und Kaninchen kann ich die Resultate der Untersuchungen von Nencki und Sieber vollkommen bestätigen.

Die Technik der Methode besteht darin, dass zuerst bei der Person, an der die Oxydationsprocesse untersucht werden sollen, das Phenol im Harn bestimmt wird. Gewöhnlich fehlt es gänzlich oder findet sich nur in Spuren. Ist aber Phenol vorhanden, so muss die Menge desselben im Laufe einiger Tage wiederholt bestimmt werden, um dann aus der Phenolmenge, die nach Einführung von Benzol in den Organismus gefunden wird, subtrahirt zu werden. Den Kranken reichte ich $1,0 \mathrm{~g}$ Benzol in Kapseln per os. Von der gesammelten Tagesmenge des Harns wurden 50 oder $100 \mathrm{ccm}$ in einen entsprechenden Kolben gebracht, mit destillirtem Wasser verdünnt und nach Zugabe von $10 \mathrm{ccm}$ $\mathrm{HCl}$ am Liebig'schen Kühler abdestillirt. Zum Destillate wurde nun Bromwasser hinzugefügt; das hierbei sich bildende Tribromphenol fällt in Form von schönen gelben Krystallen aus; diese werden auf einem vorher getrockneten und gewogenen Filter gesammelt und zwischen Filtrirpapier und darauf im Exsiccator getrocknet. Aus dem Gewicht des Filters mit dem Niederschlag wird nach Abzug des Gewichtes des trockenen Filters die Menge des Tribromphenols bestimmt. Zur Berechnung des Phenols muss die erhaltene Zahl mit 0,284 d. h. ${ }^{94} / 331$ (Moleculargewicht des Phenols und Tribromphenols) multiplicirt werden.

Zur Bestimmung der Oxydationsprocesse im Organismus existirt noch eine andere Methode, die von Prof. Poehl'1), A. Robin ${ }^{2}$ ) und Anderen vorgeschlagen wurde. Nach dieser Methode lässt sich die Energie der Oxydationsprocesse durch das Verhältniss des Stickstoffes des Harnstoffes zu der Gesammtmenge des Stickstoffes im Harn ausdrücken. Dieses Verhältniss bezeichnet Prof. Poehl als den Coefficienten

1) Grundlagen der rationellen Organotherapie von J. R. Tarobanoff, A. W. Poehl u. A. 1906, Vgl, die anderen AbhandIungen von Prof. Poehl. 2) A. Robin, Bulletin de la société médic. des hôpitaux. Fév. 1886. 
der Energie der Oxydation; letzterer beträgt bei gesunden Menschen 0,90-0,94, kann aber unter dem Einfluss verschiedener Erkrankungen bis 0,60 und sogar noch tiefer fallen. Prof. Poebl1) erwähnt, dass er seinen Gedanken von der Bedeutung des Verhältnisses des Gesammtstickstoffes zum Harnstoff schon im Jahre 1882 ausgesprochen habe, dass aber Robin unabhängig von ihm im Jahre 1886 fast zu den gleichen Resultaten gekommen sei. Die Bedeutung dieses Coefficienten wird auch von vielen anderen, besonders den französischen Autoren, wie Bouchard, Gley, Richet, Ritter u. A. ${ }^{1}$ ), bestätigt. Ich habe mich gleichfalls, sowohl bei Menschen, als auch bei Experimenten an Thieren, des Coefficienten der Energie der Athmung bedient, und kanu seine Bedeutung bestätigen.

Der Gesammtstickstoff im Harn wurde nach Kjeldahl, der Stickstoff des Harnstoffs nach Borodin bestimmt. Ich muss freilich die Mängel der letzteren Methode anerkennen, glaube aber, dass diese Methode bei den zahlreichen klinischen Untersuchungen, ihrer Einfachheit und Leichtigkeit wegen, schwerlich durch eine andere ersetzt werden könnte.

Was die Literatur über die Frage von der Giftigkeit des Harns bei gesunden und kranken Menschen anbetrifft, so ist sie zu ungeheuren Dimensionen herangewachsen. Es gehört nicht in den Rahmen dieser Arbeit, die gesammte Literatur anzuführen, und ich beschränke mich auf die Angaben, dass zuerst Segalas und Vauquelin2) schon im Jahre 1822 auf giftige Wirkung der Injection von Menschenharn in die Venen des Hundes hingewiesen haben. Feltz und Ritter ${ }^{3}$ ), Astaschewsky ${ }^{4}$ ) und viele andere Forscher bestätigten durch eine ganze Reihe von Untersuchungen die giftige Wirkung des Menschenharns. Besonders hat sich Bouchard 5 ) durch Feststellung des urotoxischen Coefficienten um die Lehre von der Giftigkeit des Harns verdient gemacht; ferner Lepine, Guerin, Hüffner, Charrin, Casciani, A. Gautier, Pouchet und viele andere, hauptsächlich französische und auch italienische Autoren. Es wurde nicht nur die Giftigkeit des Harns sichergestellt, sondern es gelang auch, giftige Substanzen aus dem Harn zu isoliren. Im Gegensatz hierzu versuchten vor Kurzem

1) Vergl. Poehl, l. c.

2) Segalas avec coll. de Vauquelin, Sur de nouvelles expériences relatives aux propriétés médicamenteuses de l'urée. Journ. de Magendie. 1822.

3) Feltz et Ritter, De l'urémie expérimentale. Paris 1881.

4) Astaschewsky, Zur Frage von der Urämie. Petersburger med. Wochenschr. 188l. No. 27.

5) Bouchard, Sur les autointoxications. Paris 1882. 
einige deutsche Autoren, Gumprechti), v. Korányi ${ }^{2}$ ), Albu ${ }^{3}$ ) und andere, dieser Meinung über die Ursache der giftigen Wirkung des Harns entgegenzutreten, indem sie nachzuweisen suchten, dass die Giftigkeit des letzteren nicht durch chemische, sondern rein physikalische Factoren bedingt wird, dank den osmotischen Erscheinungen, die beim Eindringen der hypertonischen Harnlösungen im Organismus zu Stande kommen. Die zur Klarlegung dieser wohlbegründeten Entgegnung unternommenen weiteren Versuche wiesen in der That auf die wichtige Bedeutung des osmotischen Druckes, als einer der Ursachen der Giftigkeit des Harns hin, bestätigten aber von Neuem die durch chemische Factoren bedingte Giftigkeit desselben. Bei diesen hauptsäcblich von französischen Forschern ausgefübrten Untersuchungen wurde auch die neue Methodik der Harninjectionen ausgearbeitet.

Die Theorie des osmotischen Druckes beruht auf dem Gesetze van't' Hof'f'st), nach welchem jeder lösliche Körper in seiner Lösung einen ebenso grossen Druck, in Form von osmotischem Druck, ausübt, wie derselbe Körper ihn in gastörmigem Zustande bei dem gleichen Volumen und der gleichen Temperatur auszuïben vermag. Folglich hängt der osmotische Druck von der molecularen Concentration der Lösung ab.

Die physikalische Methode der Bestimmung des osmotischen Druckes in Lösungen ist auf dem Prinzip begründet, dass die gelöste Substanz die Gefrierpunkt-Temperatur des Lösungsmittels erniedrigt. Zu meinen Bestimmungen 'der Gefrierpunkte benutzte ich den Beckmann'schen Apparat, der durch eine Wasserturbine in Bewegung gesetat wurde.

Bei der Bestimmung der Giftigkeit des Harns muss man nach den neuesten Untersuchungen von Bernard5), Joffroy und Servean ${ }^{6}$ ), und ebenso Claude Bernard und Balthazard7) die wahre oder

1) Gumprecht, Magentetanie und Autointoxication. Centralbl. f, inn. Med. 1897. No. 25.

2) v. Korányi, Physiologische und klinische Untersuchungen über den osmotischen Druck. Zeitschr. f. klin. Med. 1897. Bd. XXXIII-XXXIV.

3) Albu, Experimentelle Beiträge zur Lehre vom Harngift. Archiv f. path. Anat. u. Physiol. u. f. kilin. Med. 1901. Chemie.

4) Van't' Hoff, Vorlesungen über theoretische und physikalische

5) Bernard, Etude critique des méthodes de détermination de la toxicité du serum sanguin et de l'urine. (Revue de médec. 1900.)

6) Citirt nach Bernard.

7) Claude et Balthazard, Toxicité urinaire dans les rapports avec l'isotonie. Journal de physiologie et de pathologie générale (1900 Janvier). 
chemische Toxicität (T) und die osmotische oder physikalische Toxicität (J) unterscheiden. Beide zusammen bilden die experimentelle oder Gesammt-Toxicität (U), folglich ist die wahre Toxicität gleich der experimentellen, nach Abzug der osmotischen Toxicität: $\mathrm{T}=\mathrm{U}-\mathrm{J}$. Die weiteren Untersuchungen haben aber gezeigt, dass wir nach dieser Formel nicht die wirkliche Giftigkeit bestimmen konnen. Es musste desbalb eine Correction eingefügt werden, die durch das Fehlen der Isotonie in der Gesammttoxicität bedingt wird. Ohne auf das Nähere einzugehen, führe ich die detailirte Formel mit der Correction an: $\mathrm{T}=\mathrm{J}+\mathrm{K}(\Delta-0,56) \mathrm{U}^{2} ;(\Delta=$ Gefrierpunkt; $\mathrm{U}=$ Die Gesammttoxicität und $K=0,06$ ). Claude und Balthazard haben nach dieser Formel Correctionstabellen für die Gefrierpunkte von $-0,56$. bis - 2,00 zusammengestellt, welche die Rechnong der wahren Toxicität des gegebenen Harns mit allen Correctionen auf Grund der experimentellen Toxicität und der Gefrierpunkstsniedrigung sehr erleichtern.

Weiter stehen wir nun vor der Frage wie der Harn am besten in den Organismus des Versuchsthieres eingeführt wird; von den verschiedenen Autoren werden die mannigfaltigsten Methoden befürwortet: Durch den Mund, subcutan, in das Peritoneum, in den subarachnoidalen Raum und direct in das Blut.

Schon diese Verschiedenheit der Methoden der Einführung des. Harns zeigt, dass die son den einzelnen Autoren erhaltenen Resultate verschieden sein müssen, zumal wenu man in Betracht zieht, dass nur wenige der Forscher die Correction auf den osmotischen Druck einführten. Bouchard schlug noch im Jahre 1884 vor, den Harn in die Vena marginalis des Obres eines gewogenen Kaninchen zu injiciren. Nach Bouchard beträgt die Toxie $-\frac{\text { N. } 1000}{\text { p. }}(\mathrm{N}$ - bezeichnet die Harnmenge, die gerade im Stande ist, den Tod des gegebenen Thieres herzurufen, p - das Gewicht des Versuchsthieres) d. h. er bezeichnet als Toxie diejenige Menge des im Harn enthaltenen giftigen Prinzips, die im Stande ist ein Kilo Thier zu tödten.

Urotoxie wird die Zahl der Toxien genannt, die in der Tagesmenge des Harns vorhanden ist. Als urotoxischer Coefficient wird eine Grösse bezeichnet, die die Zahl der Toxien, die von 1 Kilo Mensch (oder Thier) im Laufe von 24 Stunden ausgeschieden werden, angiebt. Nach Bouchard beträgt der urotoxische Coefficient eines gesunden Menschen im Durchschnitt - 0,465. Diese Zabl ist aber viel zu hoch, was sich dadurch erklären lässt, dass Bouchard die experimentelle 
Ueber die Oxydationsprocesse im Organismus der Geisteskranken. 161

Toxicität für die wahre annimmt. Stefanil) brachte den Harn immer auf ein und dasselbe specifische Gewicht und fand unter Einhaltung aller sonstigen Vorsichtsmaassregeln, jedoch ohne Bestimmung der Gefrierpunktsniedrigung und ohne Correction auf den osmotischen Druck, dass der urotoxische Coefficient 0,069-0,206, im Durchschnitt also $0,13-0,15$ betrug.

Bei meinen Untersuchungen ${ }^{2}$ ) über die Giftigkeit des Harns wurde der Baru zunächst filtrirt und eine geringe Menge zur Bestimmung der Gefrierpunktsniedrigung verwendet, das Lebrige wurde in einen graduirten Zylinder mit flachen Boden gebracht, der seitlich dicht am Boden eine dünne Abflussröhre hatte. An diese Röhre wurde ein Gummischlauch, der durch eine Klemmschraube geschlossen warde und mit der hoblen Nadel endete, befestigt. Das graduirte Gefäss wurde mit einem Gummistopfen geschlossen, durch welchen eine fast bis zum Boden reichende, dünne, lange Glasröhre gesteckt wurde. Diese Vorrichtung garantirte einen gleichmässigen Druck im Gefäss. Das Gefäss mit dem Harn wurde auf einem Stativ in einer gewissen Höhe befestigt, die aber zuweilen geändert werden musste, da der Harn äusserst langsam ausströmte. Ich muss zugestehen, dass es mir trotz aller Bemühungen nicht gelang, in allen Versuchen die gleiche Schnelligkeit der Injection zu erzielen.

Der Harn wurde mit den entsprechenden Vorsichtsmaasregeln in die Ohrvene eines gewogenen Kaninchens, das an den Operationstisch mit dem Bauch nach oben gebunden war, eingeführt. Die Injection des Harns wurde mit dem letzten Athemzuge des Thieres abgebrochen.

Die von mir ausgeführten Untersuchungen betreffen 4 Gesunde und 17 Kranke, von denen. 16 an verschiedenen Geistesstörungen und eine M. R-kaja) an der Basedow'schen Krankheit litt. Weiter führe ich in aller Kürze die Angaben über die einzelnen Versuchspersonen an.

\section{Gesunde Personen.}

I. Feldscher J. W...w. Alter 35 Jahre. Gewicht 3 Pud 37 Pfund $(64,4 \mathrm{~kg})$, von niedrigem Wuchs, gesund.

1) U. Stefani. Revista sperim. di freniatr. 1900. Fasc. IV.

2) Mit den Einzelheiten der Technik der Harninjectionen und den neuesten Untersuchungen auf diesem Gebiet machte mich mein verehrter College D. J. Semeka, der sich in unserem Laboratorium mit dieser Frage beschäftigte, zuerst bekannt, wofür ich an dieser Stelle ihm nochmals meinen Dank ausspreche. 
Am 28. Januar 1903 wurden ihm per os $0,6 \mathrm{~g}$ Benzol in einer Gelatinekapsel gereicht. Am 29. Januar wurden 2350,0 com Harn rom spec. Gew. 1,015 gesammelt. Gefunden wurde Phenol (F) - 0,1895 g; Gesammtmenge des Stickstoffs im Harn der Tagesmenge $(N)-12,466 \mathrm{~g}$; Stickstoff des Harnstoffs $\left(\mathrm{N}^{0}\right)-10,555 \mathrm{~g}$. Das Verhältniss des Gesammtstickstoffes zu dem Stickstoff des Harnstoff's (Coëfficient der Energie der Oxydation nach Poehl $-0,85(85 \%)$.

Am 30. Januar wurden 3000,0 ocm Harn vom spec. Gew. 1009 ausgeschieden. Phenol-0,00341 g; an beiden Tagen zusammen also--0,193 g (Ft), oder berechnet auf $1,0=0,32$.

Am 1. Februar wurde $1,0 \mathrm{~g}$ Benzol gereicht; am 2. Februar wurden 2400,0 ccm Harn, vom spec. Gew. 1016 ausgeschieden. F-0,314.

Am 21. December 1904 betrug die Tagesmenge des Harns 2150,0 ccm, spec. Gew. 1012; Gefrierpunkt - 0,820.

Der Harn wurde in die Ohrvene eines Kaninchens von $780,0 \mathrm{~g}$ Gewicht injicirt. Das Tier verendete nach 20'. Injicirt waren $162,0 \mathrm{ccm}$ Harn.

Als Beispiel führe ich die Berechnung an:

$162,0 \mathrm{ccm}$ Harn töten $780,0 \mathrm{~g}$ Kaninchen, $1000,0 \mathrm{~g}$ Kaninchen würden von $210 \mathrm{ccm}$ Harn getötet; folglich enthalten $210 \mathrm{ccm}$ Harn eine Toxie. In $100 \mathrm{ccm}$ Harn ist 0,48 Toxie enthalten. Dieses ist die experimentelle Giftigkeit. Zur Correction muss hiervon die osmotische Toxicität abgezogen werden. Nach der Tabelle von Claudo and Balthazard ${ }^{1}$ ) entspricht dem Gefrierpunkt $-0,82$ eine osmotische Giftigkeit von $0,04+0,0156 \times 0,48^{2}=0,0436$. Nach Abzug dieser Zabl von 0,48 erhalten wir die wahre Toxicität - 0,437 in $100 \mathrm{ccm}$ Harn, und in $2150 \mathrm{cem}$ Harn um 21,5 Mal mehr, also 9,39 Toxien in der Tagesmenge,

Der urotoxische Coefficient beträgt $-\frac{9,39}{64,3}=0,146$.

Somit erhalten wir mit abgekürzten Bezeichnungen:

Ft (Phenolmenge in 24 Stunden) . . . . - - 0,31-0,32.

$\frac{\mathrm{N}^{0}}{\mathrm{~N}}$ (Oxydationscoefficient nach Poehl) . . . . - 0,85 .

Tu (Toxicität der Tagesmenge des Harns, Urotoxie) - 9,39.

Kt (urotoxischer Coëfficient) . . . . . . - 0,146.

II. Laboratoriumsdiener P. K . . . l. Alter 40 Jahre. Gewicht 5 Pud 4 Pfund $(83,43 \mathrm{~kg})$. Phenol im Harn nicht vorhanden. Von strotzender Gesundheit.

Am 12. October 1903 wurden $1460 \mathrm{ccm}$ Harn vom spec. Gew. 1023 ausschieden. $\frac{\mathrm{N}^{0}}{\mathrm{~N}}=\frac{17,37}{19,71}=0,88$. Phenol nicht vorhanden.

Erhielt 1 g Benzol.

1) Claude et Balthazard; Toxicité urinaire dans les rapports avec l'isotonie (Journal de physiologie et de pathologie générale 1900. Janvier). 
Am 13. October betrug die Tagesmenge des Harns $1200 \mathrm{ccm}$, spez. Gew. 1026. $\frac{\mathrm{N}^{0}}{\mathrm{~N}}=\frac{20,31}{18,14}=0,89 . \quad \mathrm{F}=0,34$.

14. October, $1050 \mathrm{ccm}$ Harn: spec. Gew. 1024. Phenol im Harn nicht vorhanden. Erhielt von Neuem $1 \mathrm{~g}$ Benzol. Trank viel Thee.

15. October. Es wurden $3100 \mathrm{ccm}$ Harn rom spec. Gew. 1014 ausgeschieden. $\mathrm{F}=0,36$. Die Toxicität des Harns wurde nicht bestimmt.

$$
\begin{aligned}
& \frac{\mathrm{N}^{0}}{\mathrm{~N}}=0,88-0,89 . \\
& \mathrm{Ft}=0,34-0,36 .
\end{aligned}
$$

III. Wärterin W. B ... ka. Alter 28 Jahre. Gewicht 3 Pud 35 Pfund $(63,5 \mathrm{~kg})$; leidet etwas an Kopfschmerzen.

18. December. Die Tagesmenge des Harns betrug $1350 \mathrm{ccm}$, spec. Gew. 1029. Gefrierpunkt - 2.3. Zum Injectionsversuch wurde ein Kaninchen von $1634 \mathrm{~g}$ Gewicht verwendet. Das Thier verendete nach $9^{\prime}$.

$$
\begin{aligned}
& \mathrm{UT}=(1,43-1,05) 13,5=5,13 . \\
& \mathrm{TU}=5,13 . \\
& \mathrm{Kt}=0,081 .
\end{aligned}
$$

1V. Arzt A. Ja ... ko. Alter 35 Jahre. Gewioht 4 Pud9Pfund $(69,2 \mathrm{~kg})$; gesund; Phenol im Harn nicht enthalten.

Erhielt am 9. December 1903 innerlich $1 \mathrm{~g}$ Benzol.

Am 10. December wurden $1600 \mathrm{ccm}$ Harn rom spec. Gew. 1014 ausgoschieden. $\mathrm{F}=0,31 ; \frac{\mathrm{N}^{0}}{\mathrm{~N}}=\frac{12,9}{13,8}=0,93$.

11. December. Tagesmenge des Harns - $1200 \mathrm{ccm}$; spec. Gew. - 1018; $\mathrm{F}=0$. Erhielt um $1 \mathrm{Uhr}$ Mittags von Neuem $1 \mathrm{~g}$ Benzol, und um $6 \mathrm{Uhr}$ Abends ca. $45 \mathrm{com} 40 \%$ Branntweins.

12. December. Tagesmenge des Harns $2000 \mathrm{ccm}$, spec. Gew. - 1012; $\mathrm{F}=0,202 . \frac{\mathrm{N}^{0}}{\mathrm{~N}}=\frac{12,8}{14,7}=0,39$.

11. December 1904. Gewicht 4 Pud 11 Pfond $(69,93 \mathrm{~kg})$.

Tagesmenge des Harns $1430 \mathrm{com}$; spec. Gew. 1019; Gefrierpunkt - 1,66. Ein Kaninchen ron $1050 \mathrm{~g}$ Gewicht verendete bei der Harninjection nach $1 \mathrm{~h} 10^{\prime}$. Injicirt wurde $132 \mathrm{ccm}$ Harn. Urotoxie der Tagesmenge $=(0,80-0,45) 14,3=5,0 ; \mathrm{Kt}=0,072$.

21. December. Tagesmenge des Harns $-1450 \mathrm{ccm}$; spec. Gew, -1018. Gefrierpunkt - 1,56. Ein Kaninchen von $1132 \mathrm{~g}$ Gewicht verendete nach $10^{\prime}$; $\mathrm{TU}=$ Urotoxie $=(0,96-0,4) 14,5=8,12 ; \mathrm{Kt}=0,117$.

Ft $\_0,31$ und 0,202 (nach Alkoholgenuss).

$\frac{\mathrm{N}^{0}}{\mathrm{~N}}=0,93$ und $0,89 \quad " \quad n$
$\mathrm{TU}=5,0-8,12 ; \mathrm{Kt}=0,072-0,117$. 
V. Paranoia hallucinatoria chronica et Tuberculosis pulmonum. E. J ... w. Alter 36 Jahre. Hatte als Gehülfe des Portiers in einem der Palais gedient. War am 25. November 1903 in die Anstalt übergeführt worden; wog damals 4 Pud 5 Pfund $(67,58 \mathrm{~kg})$. Trank in der letzten Zeit täglich 1/100 Wedro ${ }^{1}$ ) Branntwein und zuweilen noch mehr. Bei der Ueberführung in die Anstalt wurde die Blässe der Haut und der Schleimhäute, die Belegtheit der Zunge und die Aufgedunsenheit des Darmkanals constatirt. Die Athmung war oberflächlich, jedoch konnten in den Organen der Brusthöhle keine pathologischen Veränderungen nachgewiesen werden. Puls 88-90', Kniereflexe erhöht. Krank war E. J. seit dem Herbst 1901, als er ein furchtbares Ungehever in Gestalt eines Drachen gesehen hatte; hatte hiernach noch andere Erscheinungen und hörte viele Stimmen; begann sich als ein besonderes Wesen zu betrachten, bezog die Ereignisse der Lebensumgebung auf sich, äusserte viele Absonderlichkeiten, war aber gehorsam und blieb bis zum Tage seiner Ueberführung in die Anstalt im Dienst. In der Anstalt äusserten sich religiöse Wahnideen und Erscheinungen des Egocentrismus; er litt an Täuschungen der Sinne, besonders des Gehörs, weigerte sich, viele Gerichte, und zuweilen auch jede Speise überhaupt zu geniessen, fiel unaufhaltsam im Gewicht, sodass er während der Untersuchung der Oxydationsprozesse im September 19043 Pud 5-6 Pfund (51,2 kg) wog. Im August 1904 fing der Patient merklich zu husten an, in den Lungenspitzen wurde das Ausatmungsgeräusch hörbar, unbestimmte Athmung, Rasselgeräusche.

Stuhl nur nach Clysma. Der Patient vermied jede überflüssige Bewegung, sass lange fast unbeweglich mit geschlossenen Augen; "wenn ich mich zu bewegen beginne," ságte er, "verstärken sich die Stimmen." - "Ich bin krank," äusserte er oft, "leide aber an einer besonderen Krankheit, und kann Russland viel Trübsal und Prüfungen vorhersagen." -

Ende September trat eine Temperaturerböhung ein; es wurde Lungentuberculose constatirt; der Patient wurde in die Krankenabteilung übergeführt, wo er am 3. September 1905 an der gallopirenden Lungenschwindsucht verschied.

13. September 1904. Tagesmenge des Harns $-800 \mathrm{ccm}$; pec. Gew. 1023. Gefrierpunkt - 1,96.

Ein Kaninchen von $1270 \mathrm{~g}$ Gewicht verendete nach $9^{\prime}$.

Urotoxie $=(1,54-0,80) .8=5,9: \mathrm{kt}=0,115$.

$\frac{\mathrm{N}^{0}}{\mathrm{~N}}=\frac{12,7}{10,0}=0,78$.

Der Patient erhielt am 14. September 1 g Benzol.

15. September. Tagesmenge des Harn $-580 \mathrm{ccm}$; spec. Gew. 1025. $\mathrm{F}=0,17$.

16. September. Tagesmenge des Harns $650 \mathrm{ccm}$; spec. Gew. - 1027; $\mathrm{F}=0,038 ; \mathrm{Ft}=0,208$.

1) Eine kleine Flasche Kornbranntwein, die ungefähr $150 \mathrm{ccm} 40 \%$ Spiritus enthält. 
17. September. Tagesmenge des Ilarns $680 \mathrm{ccm}$; spec. Gew. 1025; Gefrierpunkt $=-2,17 ; \mathrm{F}=0$. Ein Kaninøben von $1740 \mathrm{~g}$ Gewicht verendete nach 10 Stunden;

Urotoxie $=(2,19-1,22) 6,8=6,6 ; \mathrm{Kt}=0,128$.

19. September. Der Patienterhielt $1 \mathrm{~g}$ Benzol.

20. September. Tagesmenge des Harns $-650 \mathrm{ccm}$; spec. Gew. - 1025; $\mathrm{F}=0,19$.

21. September. Tagesmenge des Harns $-620 \mathrm{ccm}$; spec. Gew. - 1027; $\mathrm{F}=0,04, \mathrm{Ft}=0,23$.

$$
\begin{aligned}
& \mathrm{Ft}_{\mathrm{t}}=0,208-0,23 \\
& \frac{\mathrm{N}_{0}}{\mathrm{~N}}=0,78 \\
& \mathrm{TU}=5,9-6,6 \\
& \mathrm{Kt}=0,115-0,128 .
\end{aligned}
$$

VI. Psychosis hysterica (Stadium melancholic.). M. P...wa, Mädchen. Alter 28 Jahre. Gewicht (2 Pud 20 Ffund) $40,9 \mathrm{~kg}$. Schlecht genährt. Die inneren Organe in den Grenzen der Norm; Puls 96; leicht erregbar; das Gesichtsfeld äussert unregeimässig; verengt. In Betreff der Sensibilität wurden keine besonderen Abweichungen beobachtet. Alle Reflexe erhöht; Sohluckreflex erhalten; in der Folge wurde eine starke Abschwächung desselben beobachtet. Der Vater der Kranken war an progressiver Paralyse gestorben. Sie selbst war im Alter von 17 Jahren geisteskrank. Die Krankheit äusserte sich in Verschlossenheit, Unbeweglichkeit, sinnlichen Gedanken und Weigerung der Nahrungsaufnahme. Nach zweijähriger Krankheit wurde sie, nach den Angaben der Verwandten, wieder gesund.

Zum zweiten Male erkrankte sie am Ende des Sommers 1902. Die Krankheitserscheinungen waren fast dieselben.

Während der Untersuchung der Oxydationsprocesse verhielt sich die Kranke vollkommen apathisch zu allem, und dieser Zustand wurde bisweilen nur von kurzdaaernden Zornesausbrüchen unterbrochen. Isst genügend, jedoch nur bei zwangsweiser Fütterung mit dem Löffel; täglich selbstständiger Stublgang. Schlaf befriedigend; beschäftigt sich absolut mit nichts; lilagt über nichts und antwortet auf alle Fragen entweder nichts oder ein leises "Ja" oder "Nein". Zunge nicht belegt.

Im Laufe der zweiten Hälfte des Jahres 1903 und im folgenden Jahre hatte sich ihr Zustand wenig verändert. Zuweilen traten hysterische Anfälle auf. Im August 1904 trat eine Besserung ein und die Kranke wurde von ihren Verwandten nach Hause genommen. Jetzt ist sie wieder in der Anstalt: verschlussen, erotisch.

Das Gewicht der Kranken im April 1903. 2. April 2 Pud 18 Pfund; 9. April bis 30. April 2 Pud 19 Pfund; 7. Mai 2 Pud $16^{1 / 2}$ Pfund; im August 19043 Pud $6^{1} / 2$ Pfund.

21. April 1903. Tagesmenge des Harns $650 \mathrm{ccm}$; spec. Gew. 1022; $\mathrm{F}=0,0023 ; \frac{\mathrm{N}^{0}}{\mathrm{~N}^{-}}=\frac{10,28}{6,07}=0,59$. 
22. April. Tagesmenge des Harns $500 \mathrm{ccm}$; spec. Gew. 1025; $\mathrm{F}=0,016$; $\frac{\mathrm{N}^{0}}{\mathrm{~N}}=\frac{4,05}{6,68}=0,60$. Erhielt innerlich $1 \mathrm{~g}$ Benzol.

23. April. Tagesmenge des Harns $1100 \mathrm{ccm}$; spec. Gew. $1013 ; \mathrm{F}=0,266$.

24. April. Tagesmenge des Harns $350 \mathrm{ccm}$; spec. Gew. 1024; $\mathrm{F}=0,045$.

25. April. Tagesmenge des Harns $500 \mathrm{ccm}$; spec. Gew. 1025; $\mathrm{F}=0,002$. $\mathrm{Ft}=0,311-0,04=0,307$.

1. Mai. Im Harn nur Spuren von Phenol. Erhielt $1 \mathrm{~g}$ Benzol.

2. Mai. Tagesmenge des Harns $1080 \mathrm{ccm}$; spec. Gew. 1014; $\mathrm{F}=0,25$.

3. Mai. Tagesmenge des Harns $950 \mathrm{ccm}$; spec. Gew. 1017; $\mathrm{F}=0,033$.

4. Mai. Tagesmenge des Harns $600 \mathrm{ccm}$; spec. Gew. 1014; $\mathrm{F}=$ Spuren An Abend begannen die Menses.

$\mathrm{Ft}=0,28$.

$$
\begin{aligned}
& \mathrm{Ft}=0,307-0,28 \\
& \frac{\mathrm{N}^{0}}{\mathrm{~N}}=0,59-0,60
\end{aligned}
$$

VII. Melancholia periodica. Ja. J . . . w. Alter 59 Jahre. Wurde am 28. November 1903 in die Anstalt übergeführt. Kaufmann. Gewicht (5 Pud 17 Pfund) $=88,75 \mathrm{~kg}$. Ernährungszust nd genügend. Pupillen gleichmässig, reagiren gut; Zunge leicht nach links gerichtet, zittert, hat die charakteristische

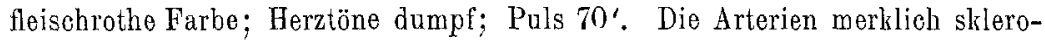
tisirt; Reflexe erniedrigt; hatte im Jahre 1895 eine ähnliche Geisteskrankheit überstanden, wie die jetzige, und war damals nach 2 Monaten genesen. Jetzt hatte die Krankheit vor zwei Monaten begonnen und ausserte sich in Schlaflosigkeit, drückendem Gefühl in der Herzgegend und trauriger Gemüthsstimmung. An den ersten zwei Tagen nach der Ueberführung in die Anstalt trat besonders die traurige Stimmung, das erniedrigte Selbstbefinden und eine äusserste Unentschlossenheit hervor. Leidender Gesichtsausdruck, leise und langsame Sprache. Kopfschmerzen, Verstopfungen. Nach Reinigung und Desinfection des Darmkanals, beruhigenden Mitteln und längeren, warmen Bädern begann das Befinden des Kranken sich rasch zu bessern; am 5. December ist in der Krankheitsgeschichte bereits bemerkt, dass der Patient viel im Freien spazieren geht und Billard spielt. Gemüthsstimmung und Selbstbefinden befriedigend; schläft besser; selbstständiger Stuhlgang. Wurde am 10. December von seiner Frau auf Urlaub genommen, kehrte am 16. December in die Anstalt zurück, um sich vom Arzt untersuchen zu lassen und verliess sie am selben Tage psychiseb gesund.

:Gewicht am 26. November (5 Pud 17 Pfund) 88,75 kg; 3. December (5 Pud 20 Pfund) $89,98 \mathrm{~kg}$; am 10. December (5 Pud 22 Pfund) $90,79 \mathrm{~kg}$.

30. November. Tagesmenge des Harns $1500 \mathrm{ccm}$; spec. Gew. 1012. Erbielt innerlioh $1 \mathrm{~g}$ Benzol.

1. December. Tagesmenge des Harns $1620 \mathrm{ccm}$; spec. Gew. 1012; $\mathrm{F}=0,203 ; \frac{\mathrm{N} 0}{\mathrm{~N}}=\frac{8,7}{9,96}=0,87$. 
2. December. Tagesmenge des Harns $1900 \mathrm{ccm}$; spec. Gew. 1010; $\mathrm{F}=0,011 ; \mathrm{Ft}=0,214$.

5. December. Phenol im Harn nicht vorhanden. Erhielt $1 \mathrm{~g}$ Benzol.

6. December. Tagesmenge des Harns 1600 cem; spec. Gew. 1011; $\mathrm{F}=0,338 ; \frac{\mathrm{N}^{0}}{\mathrm{~N}}=\frac{9,36}{10,6}=0,88$.

7. December. Phenol im Harn nicht vorhanden.

Während der Krankheit:

$$
\begin{aligned}
& \frac{\mathrm{Ft}}{\overline{\mathrm{N}^{0}}}=0,214 \\
& \mathrm{~N}=0,87
\end{aligned}
$$

Nach der Genesung:

$$
\begin{aligned}
& \mathrm{Ft}=0,338 \\
& \frac{\mathrm{N}^{0}}{\mathrm{~N}}=0,88
\end{aligned}
$$

VIII. Melancholia. E. B. . . wa. Unverehe]icht. Alter 47 Jahre. Trat am 14. Juli 1902 in die Anstalt. Gewicht (3 Pud 5 Pfund) $51,12 \mathrm{~kg}$. Sie stammt aus einer degenerativen Familie. Die Anzeichen der Geistesstörung begannen sich im April 1902 zu äussern und fielen mit dem Beginn der klimakterischen Periode zusammen. Im Februar 1903, als die ersten Untersuchungen der Oxydationsprocesse unternommen wurden, herrschte bei der Kranken eine äusserst finstere Stimmung und erniedrigtes Selbstbefinden vor; sie ächzt oft und äussert einförmige Wahnideen von Selbstbeschuldigungen und sinnliche Gedanken. Schläft schlecht; springt oft vom Bett auf und läuft in der Abtheilung umber. Zunge trocken und belegt; unangenehmer Geruch aus dem Munde. Stuhl täglich, jedoch nur nach Clysma und Abführungsmitteln. Isst sehr wenig, und hörte am 7. Februar gänzlich auf Speise zu sich zu nehmen, so dass zur künstlichen Ernährung geschritten werden musste; die Ration bestand aus 3 Eiern, 3 Glas Milch, $30 \mathrm{~g}$ Brodpulver, $30 \mathrm{~g}$ Fleischpulver und $2 \mathrm{ccm}$ Acid. mur. dil. Puls 88-94. Am 13. Februar wurden subcutane Injectionen von physiologischer Kochsalzlösung zu je $800 \mathrm{ccm}$ begonnen, wonach eine merkliche Besserung eintrat; am 18. Februar begann die Kranke selbst zu essen. Vom 8. März an wurdo in Folge der Klagen der Kranken über die Schmerzen bei den Injectionen and des Widerstandes die Flüssigkeitsmenge bis auf $500 \mathrm{ccm}$ erniedrigt; am 5. April wurden die Injectionen gänzlich eingestellt. Bis zum 20. April war das Befinden der Kranken etwas besser und besserte sich gegen Ende des Monats noch mehr, obgleich die Gemüthstimmung eine unruhige blieb, und Erscheinungen des Negativismus und zeitweise aggressive Handlungen auftraten. Der melancholische Zustand währte bis zum Herbst 1906, als unerwartet eine Erregung eintrat, die bis jetzt noch andauert. Gewicht beim Eintritt in die Anstalt 3 Pud 5 Pfund.

12. Februar 19032 Pud 20 Pfd. 9. April 2 Pud 32 Pfd. und 23. April 2 Pad $35^{1 / 2} \mathrm{Pfd}$.

7. Februar. Tagesmenge des Harns $1050 \mathrm{ccm}$; spec. Gew. 1023; $\mathrm{F}=0,012$; $\frac{\mathrm{N}^{0}}{\mathrm{~N}}=\frac{9,817}{14,7}=0,66$. Erhielt innerlich $1 \mathrm{~g}$ Benzol. 
8. Februar. Tagesmenge des Harns $650 \mathrm{ccm}$; spec. Gew. 1019; $\mathrm{F}=0,277$;

$$
\frac{\mathrm{N}^{0}}{\mathrm{~N}}=\frac{5,05}{7,08}=0,7.1 \text {. }
$$

9. Februar. Tagesmenge des Harns $640 \mathrm{~cm}$; spec. Gew. 1016; $\mathrm{F}=0,032$.

10. Februar. Tagesmenge des Harns $580 \mathrm{ccm}$; sp. Gew. 1018; $\mathrm{F}=0,027$;

$$
\frac{\mathrm{N}^{0}}{\mathrm{~N}}=\frac{3,94}{5,48}=0,72 \text {. }
$$

11. Februar. Der Harn konnte vicht gesammelt werden.

12. Februar. Tagesmenge des Harns $720 \mathrm{~cm}$; sp. Gew. $1017 ; \mathrm{F}=0,013$;

$$
\mathrm{Ft}=0,3 \text {. }
$$

15. April 1903. Tagesmenge des Harns $1750 \mathrm{~cm}$; sp. Gew. 1011; $\mathrm{F}=0$;

$$
\frac{\mathrm{N}^{0}}{\mathrm{~N}}=\frac{7,25}{9,03}=0,80 \text {. }
$$

16. April. Tagesmenge des Harns 1800; spec. Gew. 1011; $F=0$; $\frac{\mathrm{N}^{0}}{\mathrm{~N}}=\frac{7,91}{9,23}=0,85$. Erhält innerlich $1 \mathrm{~g} \mathrm{Benzol}$.

17. April. Tagesmenge des Harns $1350 \mathrm{com}$; spec. Gew. $1013 ; \mathrm{F}=0,324$.

18. April. Tagesmenge des Harns $1350 \mathrm{ccm}$; spec. Gew. 1012; $\mathrm{F}=0,041$.

19. April. Spuren von Phenol fit $=0,365$.

$$
\begin{aligned}
& \mathrm{Ft}=0,3 \quad \text { Nach subcutanen Injec- } \quad \mathrm{Ft}=0,365
\end{aligned}
$$

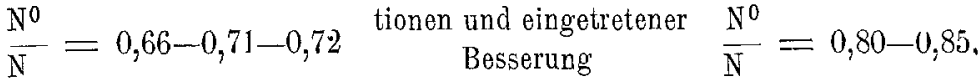

IX. Stadium melancholicum psychosis circularis. Unverehelichte A. K .. wa. Alter 52 Jahre. Wurde am 14. April 1905 in die Anstalt aufgenommen. Hatte zum ersten Mal schon in der Jugend eine geistige Erkrankung überstanden; stammt aus einer äusserst degenerirten Familie. $Z_{w i s c h e n}$ der ersten und zweiten und ebenso $z$ wischen der $z$ weiten und dritten Erkrankung waren vollkommen freie Intervalle gewesen. Die dritte Erkrankung im Jahre 1892 hatte vier Monate gedauert; hiernach war ein freies Intervall bis zum Jahre 1898 eingetreten; vom 24 . Juli 1898 bis zum 1. August 1900 befand sie sich in der Irrenanstalt. Zum zweiten Male blieb sie rom 7. August 1904 bis zum 6. März 1905 in der Anstalt. Die Krankheit äusserte sich in Anfällen von maniakalischer Erregung und Verwirrung, dann folgten freie Intervalle und dann ein melancholischer Zustand mit Wahnideen von sinnlichen Gedanken und Verbrechen, Verzweiflung usw. Während der freien Intervalle tritt jetzt eine allgemeine Schwächung des Urtheils, des Willens und des Denkvermögens, Hast, leichte Reizbarkeit und dem Aehnliches auf. Seit jhrer Ueberführung in die Anstalt am 14. Angust 1905 bis zum September hatte die Kranke einen Anfall von maniakalischer Erregung überstanden; sie sang, schrie, tanzte, stampfte usw. Mitte September trat ein freies Intervall ein; während dieser Zeit las die Kranke, arbeitete, ging viel spaziren und nutzte ihre Freiheit aus; oft klagte sie nur über Kopfschmerzen, besonders während 
der Menses. Zeitweilig wurde ejn Anschwellen ihrer Handgelenke auf arthritischem Boden beobachtet.

Am 9. September 1906 wurde die Kranke bei gutem Befinden auf Urlaub entlassen, wurde aber am 26. September in schwer deprimirtem Zustande in die Anstalt zurückgebracht: das Gesicht - maskenartig; Darmkanal überfüllt; verwejgert jede Speiseaufnahme; beantwortet nicht die Fragen, und wiederholt beständig im. Flüsterton: "Ich unglückliche, ach mein Gott." Es mussten ihr ungeheure Mengen von Abführungsmitteln gegeben werden, verschiedenartige Clysmen gemacht werden und zuweilen musste sie durch die Sonde genährt werden. Dieser Zustand dauerte bis zur zweiten Hälfte des Octobers, als mit der Wiederherstellung der selbständigen Thätigkeit des Darms eine Besserung des psychischen Befindens eintrat; obgleich die Kranke ibre Wahnideen von von Sünden und Gebeten wiederholt äusserte, begann sie gut zu essen, ging spaziren, beschäftigte sich sogar mit Handarbeit und zeigte Interesse für das umgebende Leben. Am 10. - 15. November kam die schnell fortschreitende Besserung zum Stillstand und ihr Zustand verschlechterte sich eher.

Gewicht am 11. Oktober 3 Pud 17 Pf. $(56,0 \mathrm{~kg}) ; 20$. Oktober 3 Pud 20Pf. $(57,26 \mathrm{~kg}) ; 1$. November 3 Pud 9 Pf. $(52,76 \mathrm{~kg}) ; 15$. November 3 Pud 11 Pf. $(53,57 \mathrm{~kg}) ; 22$. November 3 Pud $12 \mathrm{Pf}$. (53.98 lg).

11. October 1906. Tagesmenge des Harns $880 \mathrm{ccm}$; spec. Gew. 1023; Gefrierpunkt - 1,42; ein Kaninchen ron $2665 \mathrm{~g}$ Gewicht verendete nach $35 \mathrm{Min}$. $\mathrm{TU}=(1,9-0,466) 8,8=12,62$; Im Harn Spuren von Phenol.

21. October. Durch die Sonde wird $1 \mathrm{~g}$ Benzol eingeführt.

22. October. Tagesmenge des Harns $950 \mathrm{ccm}$; spec. Gew. 1016; $\mathrm{F}=0,26$. (Es ist möglich, dass nicht alles Benzol in den Magen gelangt war, und ein Theil an den Wandungen der Sonde haften geblieben war, oder dass ein Theil des Harns beim Stuhlgang verloren gegangen ist!)

23. October. Im Harn Spuren von Phenol.

31. October. Gesammtmenge des Harns $800 \mathrm{ccm}$; Gefrierpunkt $-1,23$; Ein Kaninchen ron $1300 \mathrm{~g}$ Gewicht verendete nach $20 \mathrm{Min}$. $\mathrm{TU}=(0,61-0,2)$ $8=3,28$.

2. November. Gesammtmonge des Harns $870 \mathrm{ccm}$; spec. Gew. 1018; Gefrierpunkt - 1,19. Ein Kaninchen von $1390 \mathrm{~g}$ Gewicht verendete nach $40 \mathrm{Min}$. $\mathrm{TU}=(0,6-0,18)=3,65$.

15. November. Erhielt $1 \mathrm{~g}$ Benzol per os.

16. November. Harnmenge für 26 Stunden $1500 \mathrm{ccm}$; spec. Gew. 1014. $\mathrm{F}=0,51$.

23. November. Tagesmenge des Harns $1200 \mathrm{ccm}$; spec. Gew. 1019; Gefrierpunkt - 1,36. Ein Kaninchen von $990 \mathrm{~g}$ Gewicht verendete nach $40 \mathrm{Min}$. $\mathrm{TU}=9,14$.

Bei schlechtem Befinden

$$
\begin{array}{rl}
\mathrm{Tt}=0,26 & \mathrm{Ft}=0,51 \\
\mathrm{Tu}=12,62 & \mathrm{Tu}=3,28-3,65-9,14(!) \\
\mathrm{Kt}=0,22 & \mathrm{Kt}=0,07-0,061-0,172(!)
\end{array}
$$

Bei einer gewissen Besserung 
X. Katatonia. W. N ... W. Wurde am 20. April 1903 in die Anstalt übergeführt. Student; stammt aus einer degenerirten Familie. Seine gedrückte Stimmung und seine Absonderlichkeiten wurden schon im Frühling 1902 bemerlit; augenscheinliche Anzeichen der Geistesstörung traten aber erst vor Kurzem auf. Schlecht genährt, etwas blutarm; Gesichtsausdruck unbeweglichmaskenartig; Pupillen erweitert; eine gewisse Anschwellung der Gland. thyreoideae; die Zunge zittert; Puls 80; Kniereflexe abgeschwächt, Unterleibsreflexe fehlen; Gremastera und vasomotorische Reflexe verstärkt; Zittern in den Fingern.

In den ersten Tagen sagt er leise, dass er schwer bedrückt ist, dass er ernstlich krank ist; er hört Stimmen, wessen, kann er jedoch nicht angeben; die Bewegungen sind schlaff und unentschlossen; interessirt sioh absolut für nichts und beschäftigt sich mit nichts. Im Mai 1903 wurde der Zustand des Patienten folgendermaassen beschrieben: Bowegungen schlaff und unentschlossen, das Gesicht maskenartig; vollkommene Gleichgültigkeit zur Umgebung; unbequeme, gezwungene Stellungen. Auf Fragen antwortet er entweder nicht, oder es gelingt nur mit Mühe ihm ein "Ja" oder "Nein" abzunöthigen. Wird mit dem Löffel genährt, isst aber im ganzen genügend. Kurz danach traten Erscheinungen des Negativismus und der Katalepsie auf. Wurde am 30. Mai 1904 von seiner Mutter aus der Anstalt genommen.

Gewicht 7. Mai 3 Pud $25^{1 / 2}$ Pf. $(59,35 \mathrm{lg}) ; 28$. Mai $3 \mathrm{Pud} \mathrm{221/4} \mathrm{Pf}$. $(58,17 \mathrm{~kg})$.

2. Mai 1903. Tagesmenge des Harns 1300 ccm.; spec. Gew. 1014; $\mathrm{F}=0,002 \frac{\mathrm{N}^{0}}{\mathrm{~N}}=\frac{9,8}{11,29}=0,86$.

3. Mai. Erhielt $1 \mathrm{~g}$ Benzol.

4. Mai. Tagesmenge des Harns $1100 \mathrm{ccm}$; spec. Gew. 1023; $\mathrm{F}=0,369$; $\frac{\mathrm{N}^{0}}{\mathrm{~N}}=\frac{11,34}{13,09}=0,85$.

5. Mai. Tagesmenge des Harns $1200 \mathrm{ccm}$; spec. Gew. 1021; $\mathrm{F}=0,01$; $\mathrm{Ft}=0,379$.

6. Mai. Spuren von Phenol in Harn.

7. Mai. Erhielt $1 \mathrm{~g}$ Benzol.

8. Mai. Tagesmenge des Harns $1150 \mathrm{ccm}$; speo. Gew. 1016; $\mathrm{F}=0,367$.

9. Mai. Tagesmenge des Harns $1300 \mathrm{ccm}$; spec. Gew. 1018; Spuren von Phenol.

$$
\mathrm{Ft} .=0,367-0,379
$$

$$
\frac{\mathrm{N}^{0}}{\mathrm{~N}}=0,86-0,85 \text {. }
$$

XI. Katatonia. B. K. . . ge. Alter 18 Jahre; Student. Trat am 4. Juli 1904 in das Krankenhaus. Wog zu dieser Zeit 2 Pud 36 Pf. $(47,44 \mathrm{~kg})$. Von schwachem Körperbau und schlecht genährt. Blässe der Haut; auf dem Körper viele Acne vulgaris; die Lymphdrüsen lassen sich nicht hindurchfühlen; der Hals im Gebiete der Gland. thyreoideae merklich geschwollen. Die Augen sind geschlossen und der Patient widersetzt sich dem Versuch sie zu öffnen. Zunge 
trocken. Hunger; Geruch aus dem Munde; das Zahnfleisch blutend. Athembewegungen sehr oberflächlich. Herztöne rein; Puls 80 , verstärkt sich schnell bei der geringsten Erregung des Kranken. Auf der Haut erscheint und verschwindet ohne jeden äusseren Grund die eryethmatöse Röthe. Hautreflexe verstärkt; Gelenkreflexe konnten nicht bervorgerufen werden, da die Muskeln gespannt sind. Liegt im Bett, wird durch die Sonde ernährt; Unreinlich. Leidet an Verstopfungen; während der Untersuchung der Oxydationsprocesse war in Betreff des Kranken vermerkt: liegt ohne jede Bewegung, mit geschlossenen Augen im Bet:; reagirt nicht auf die Umgebung. Während der Ernährung durch die Sonde setzt er einen passiven Widerstand entgegen. Zeitweise treten kataleptoide Erscheinungen auf. Stuhl nur mit Clysma. Wird durch die Sonde ernährt, unreinlich. Erhebt sich zuweilen und fixirt scharf irgend einen Punkt. Gewicht im September 19042 Pud 28 Pf. $(44,2 \mathrm{~kg})$. Dieser Zustand währte die ganze Zeit mit geringen Veränderungen, indem der Patient der Fütterung einen activen Widerstand entgegensetzte, schimpfte und drohte. Oft endete die Fütterung mit Erbrechen; der Magen musste nicht selten gereinigt werden. War während der ganzen Zeit unreinlich; ein soleher Zustand dauerte bis zum Ende October, als eine merkliche Besserung eintrat. Der Patient wusch sich selbst im Bett, ging, wenn auch unbekleidet, vom Bett bis zur Wanne durch das Zimmer. Vom 2. November an begann er selbständig und in genügendem Maasse zu essen, jedoch nur vegetabilisehe Kost; begann sich anzukleiden. Schreibt viel, ist jetzt immer angekleidet; gegen alle höflich. Isst selbst und schreibt den ganzen Tag; beschreibt ausfübrlich, was um ihn her vorgeht, äussert aber viele sonderbare und hypochondrische Ideen. Der pbysische Zustand bessert sich sichtlich. Worde in die ruhige Abtheilung übergeführt.

Gewicht im 0etober 19062 Pud $4-2$ Pud 5 Pf. (34 kg). Weigerte sich im November sich wiegen zu lassen.

30. August 1904. Tagesmenge des Harns $1000 \mathrm{ccm}$; spec. Gew. 1017; Gefrierpunlit $-1,43$. $\mathrm{TU}=(1,25-0,37) 10=8,8$.

31. August. Erhielt $1 \mathrm{~g}$ Benzol.

1. September. Tagesmenge des Harns $1550 \mathrm{ccm}$; speo. Gew. 1011; $\frac{\mathrm{N}^{\mathrm{O}}}{\mathrm{N}}=\frac{10}{11,22}=0,89 ; \mathrm{F}=0,462$.

2. September. Tagesmenge des Harns $1400 \mathrm{ccm}$; spec. Gew. 1014; $\mathrm{F}=0,004 . \mathrm{Ft}=0,466$.

3. September. Tagesmenge des Harns $1600 \mathrm{ccm}$; spec. Gew. 1012; Spuren von Phenol.

4. September. Tagesmenge des Harns $1150 \mathrm{ccm}$; spec. Gew. 1015; Gefrierpunkt $-1,22 ; \frac{\mathrm{N}^{0}}{\mathrm{~N}}=\frac{1077}{12,23}=0,82 ; \mathrm{TU}=(1,0-0,219) 11,5=8,98$.

7. September. Durch die Sonde wurde $1 \mathrm{~g}$ Benzol eingeführt.

8. September. Tagesmenge des Harns $2100 \mathrm{ccm} ; \mathrm{F}=0,233$. 
9. September. Tagesmenge des Harns $1800 \mathrm{ccm}$; spec. Gew. 1013; $\mathrm{F}=0,174 ; \mathrm{Ft}=0,407$. Während der Besserung im Befinden.

14. September 1906. Tagesmenge des Harns $1250 \mathrm{ccm}$; spec. Gew. 1017; Gefrierpunkt - 1,34; Ein Kaninchen von $1100 \mathrm{~g}$ Gewicht vorendete nach 11 Min. $\mathrm{TU}=(0,43-0,24) 12,5=2,37$. Im Harn Spuren von Pheriol.

15. Norember. Erhielt 1 g Benzol.

16. November. Tagesmenge des Harns $2100 \mathrm{ccm}$; spec. Gew. 1011; $F=0,31$.

18. November. Tagesmenge des Harns 750 cem; spec. Gew. 1019; Gefrierpunkt - 1,35. Ein Kaninchen ron $1078 \mathrm{~g}$ Gewicht verendete rach $23 \mathrm{Min}$; $\mathrm{TU}=(0,5-0,25) 75=1,87$.

$$
\begin{aligned}
& \text { Bei schwerem Zustande } \\
& \mathrm{Ft}=0,466-0,407 \\
& \frac{\mathrm{N}_{0}}{\mathrm{~N}}=0,89-0,82 \\
& \mathrm{TU}=8,8-8,98 \\
& \mathrm{Kt}=0,2-0,204
\end{aligned}
$$

Während der Besserung

$\mathrm{Ft}=0,31$

$$
\begin{aligned}
& \mathrm{TU}=2,37-1,87 \\
& \mathrm{Kt}=0,07-0,055
\end{aligned}
$$

XII. Paranoia chronica (Dementia paranoides). P. S...n. Alter 25 Jahre, Geistlicher; trat am 19. Februar 1903 in die Anstalt. Gewicht 2 Pud 38 Pf. $(48,20 \mathrm{~kg})$. Von schwachem Körperbau; schlecht genährt; Haut blass, Knie-, Unterleibs- und vasomotorisehe Reflexe erhöht, die am Cremaster abgeschwächt. Herztöne etwas gedämpft; Puls 80; Temperatur gewöhnlich, ca. 370. Eine leichto Anschwellung im Gebiet der Glandula thyreoidea. Eine ausgesprochene Geistesstörung hatte sich erst vor Kurzem gezeigt; nach den Worten des Bruders waren aber schon früher "Absonderlichleiten und annormales Benehmen" bemerkt worden. Während der letzten 2 Jahre hatte er $4 \mathrm{Mal}$ seine Stelle gewechselt, wobei es ihm überall schien, dass man sich feindlich gegen ihn verhalte und ihn wegen irgend etwas im Verdacht habe. Als or in die Anstalt gebracht wurde, war er äusserst unruhig, wollte nicht im Bett liegen, versuchte aufzuspringen, griff den umgebenden Personen nach den Händen erhob die Hände nach oben, griff sich in die Haare, bat ihn gehen zu lassen;- schläft schlecht und isst wenig. Als er sich beruhigt hatte, erklärt er, dass er wisse, wo er siuh befinde, sagt, dass er einförmige Stimmen höre, die wiederholen "Genug, ertränken, Closet", und dem ähnliches, diese Worte würden ihm von Kosaken zugeschrieen. Selbst hält er sich für vollkommen gesund. Im Laufe der ganzen Zeit äusserte der Patient wenig Interesse für die Umgebung, lebte mehr im Gebiete seiner Sinnestäuschungen und Wahnvorstellungen; Gemüthsstimmung veränderlich, meistens jedoch finster und böse. Selbst sagt der Kranke nicht selten, dass es ihm wirr im Kopfe sei und er keine Gedankèn habe. Gewöhnlich wiederholt er stereotypisch „Ieh hin gesund; geben Sie mir mein Kreuz und mein Evangelium wieder"s und er wendet sich an Alle mit dieser Bitte. Aeussert unsystematische Verfolgungsideen; und begeht viele unmotivirte Handlungen. Wurde von seinem Bruder am 2. Angust 1904 aus der Anstalt genommen. 
Gewicht 26. Februar 19032 Pud 39 Pfund. 5. März 3 Pud 1 Pfund. 19. März 2 Pud 38 Pfund.

22. Februar 1903. Tagesmenge des Harns $650 \mathrm{ccm}$; spec. Gew. 1021; $\mathrm{F}=0,006$. Erhielt $1 \mathrm{~g}$ Benzol.

23. Februar. Tagesmenge des Harns $860 \mathrm{ccm} ; \mathrm{F}=0,466$.

24. Februar. Tagesmenge des Harns $800 \mathrm{ccm} ; \mathrm{F}=0,015$.

25. Februar. Tagesmenge des Harns $1600 \mathrm{ccm} ; F=0,01$. Tt $=0,491$ $-0,018=0,473$.

1. März. Im Harn Spuren von Phenol; erhielt $1 \mathrm{~g}$ Benzol.

2. März. Tagesmenge des Harns $580 \mathrm{ccm}$; spec. Gew. 1026; F $=0,591$.

3. März. Tagesmenge des Harns $700 \mathrm{cem} ; \mathrm{F}=0,004 ; \mathrm{Ft}=0,595$.

5. März. Tagesmenge des Harns $900 \mathrm{ccm} ; \frac{\mathrm{N}^{0}}{\mathrm{~N}}=\frac{10,27}{12,89}=0,79$.

6. März. Tagesmenge des Harns $1400 \mathrm{ccm} ; \frac{\mathrm{N}^{0}}{\mathrm{~N}}=\frac{8,95}{10,92}=0,81$.

$$
\mathrm{Tt}=0,473-0,595 \quad \frac{\mathrm{N}^{0}}{\mathrm{~N}}=0,79-0,81 .
$$

XIII. Paranoia chronica (Dementia paranoides). B....w. Officier. Alter 25 Jahre. Wurde am 17. November 1903 in die Anstalt übergeführt. Gewicht (4 Pud 8 Pfand) $68,7 \mathrm{~kg}$. Ziemlich gut gebaut und ernährt. Die Zunge zittert ein wenig; Puls $80-120$, unter dem Finfluss der geringsten Gemüthsbewegungen leicht erregbar. Geschlechtstrieb abgeschwächt; Knie-, Hacken und vasomotorische Reflexe verstärkt, Cremastera abgeschwächt; stammt aus einer stark degenerirten Familie. Die Anzeichen der geistigen Störung entwickelten sich allmählich vom Jahre 1900 an. Zuerst trat Reizbarkeit und veränderliche Gemüthsstimmung auf, nder Wunsch Jemand zu retten, mit Jemand zu kämpfen", weiter die Erscheinungen des Egocentrismus; der Kranke begann das Husten und die Gesten der ihn umgebenden Personen auf sich zu beziehen; es erfolgten heftige Auftritte mit anderen Personen und Erklärungen mit der Polizei. Der Kranke musste in eine speciolle Heilanstalt gebracht worden, aus welcher ihn seine Mutter zu sich nahm. Nachdem er in die Irren: anstalt übergeführt worden war, zeigten sich in der ersten Zeit Erscheinungen des Egocentrismus and der Symbolisation, und Gehörstänschungen. Der Patient war äusserst leicht erregbar und schlug sich sogar. Im Laufe der Zeit liessen die Erscheinungen des Egocentrismus, Wahnideen und Sinnestäuschungen

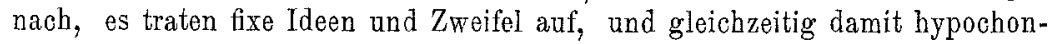
drische Ideen und eine merkliche Abschwächung der Psyche überhaupt.

Verliess am 13. Mai 1904 die Anstalt im Zustande der Besserung. Das Gewicht schwankte zwischen 4 Pud 7 Pfund und 4 Pad 9 Pfund.

17. Deoember 1903. Phenol im Harn nicht vorhanden. Erhielt $1 \mathrm{~g}$ Benzol.

18. December. Tagesmenge des Harns $2200 \mathrm{ccm}$; spec. Gew. 1015, $F=0,34 ; \frac{N^{0}}{\mathrm{~N}}=\frac{15,92}{18, \overline{97}}=0,83$. 
19. December. Tagesmenge des Harns $2250 \mathrm{ccm}$; spec. Gew. 1017; $\mathrm{F}=0,04 . \mathrm{Ft}=0,38$. Erhielt $1 \mathrm{~g}$ Benzol.

20. December. Tagesmenge des Harns $2400 \mathrm{ccm}$; spec. Gew. 1017; $\mathrm{F}=0,367$.

21. December. Tagesmenge des Harns $2200 \mathrm{ccm}$; spec. Gew. 1017; $\mathrm{F}=0,06 ; \frac{\mathrm{N}^{0}}{\mathrm{~N}}=\frac{16,26}{19,13}=0,84$.

$$
\text { Ft }=0,38-0,427 \quad \frac{\mathrm{N}^{0}}{\mathrm{~N}}=0,83-0,84 .
$$

XIV. Psychosis degenerat. (Dementia paranoides). A. S., w. Alter 28 Jahre. Betrat die Anstalt am 16. December 1901. Gewicht (3 Pud $25 \frac{1}{2}$ Pfund) $71,54 \mathrm{~kg}$. Erkrankte, als er noch Student der Universität war, ungefähr im Jahre 1897. Die Krankheit begann mit Apathie, Arbeitsunfähigkeit, unbegründete Unruhe, dem Gefühl der physischen Schwäche und hypochondrischen und fixen Ideen. Während der Untersuchungen des Harns im Jahre 1903 und 1904 war der Zustand des Patienten folgendermaassen oharakterisirt worden: Pupillen merklich erweitert, Zunge stark belegt, Puls leicht erregbar, 84'-90'; Sensibilität in allen Hinsichten normal; Sehnen- und Muskelreflexe äusserst verschärft; Dermographismus, Reflex des Cremasters abgeschwächt. Jederlei Arbeit fällt dem Patienten äusserst schwer, und er wejgert sich unter den allermöglichsten Vorwänden etwas zu thun; klagt über leichte Ermüdbarkeit (Koschmar) und nicht erquickenden Schlaf, vor Allem aber über die beständige Gespanntheit. "Angeschwollenheit" der Gesichtsmuskeln. Nach der Meinung des Patienten verräth ihn diese Angeschwollenheit, offenbart seinen Zustand und lenkt die allgemeine Aufmerksamkeit auf ihn, weshalb er auch die Menschen meidet. Der Kranke wendet sich beständig an alle Aerzte mit hypochondrischen Klagen anlässlich der verschiedenartigsten Symptome betreffs Magen, Herz, Gedächtniss, Willen, Combinationsvermögen etc. Angst vor den verschiedenartigsten Dingen, fixe Ideen, Zweifel, Erscheinungen des Negativismus und Egocentrismus: der Kranke liebt zu baden; der Arzt braucht aber nur zu sagen, dass ihm die Bäder wohl bekommen, so wird er lange Zeit das Entgegengesetzte zu beweisen suchen und an dem betreffenden Tage kein Bad nehmen. Nichts bedeutende Worte und Bewegungen der umgebenden Personen bezieht er von Zeit za Zeit auf sich. Es werden auch sprunghafte Wahnideen von Verfolgung und Vergiftung beobachtet. Isst und schläft ungeachtet aller Klagen gut, täglich selbstständiger Stuhlgang. Gewicht ca. 3 Pud 25 Pfund bis 3 Pud 28 Pfund (ca. $60 \mathrm{~kg}$ ). Der Kranke befindet sich gegenwärtig in der Anstalt. Die psychische Anomalie tritt noch schärfer hervor. Es treten sogar monomanische Handlungen und gezwungene Stellungen auf.

22. März. Tagesmenge des Harns $1360 \mathrm{ccm}$; spec. Gew. 1017; Phenol fehlt; $\frac{\mathrm{N}^{0}}{\mathrm{~N}}=\frac{11,78}{13,88}=0,85$.

24. März. Erhielt 1 g Benzol. 
25. März. Tagesmenge des Harns $1000 \mathrm{ccm}$; speo. Gew. 1025; $\mathrm{F}=0,552$.

26. März. Tagesmenge des Harns $1750 \mathrm{ccm}$; spee. Gew. 1017; unwägbare Spuren von Phenol.

29. März. Erbielt $1 \mathrm{~g}$ Benzol.

30. März. Tagesmenge des Harns $1400 \mathrm{ccm}$; spec. Gew. $1018 ; \mathrm{F}=0,466$.

31. März. Tagesmenge des Harns $1400 \mathrm{ccm}$; spec. Gew. 1018. Spuren von Phenol.

12. September 1904. Tagesmenge des Harns $1000 \mathrm{ccm}$; spec. Gew. 1022; Gefrierpunkt $=-2,0$. Ein Kaninchen von $1250 \mathrm{~g}$ Gewioht verendete nach 16 Stunden. Injicirt wurden $55 \mathrm{com}$ Harn, $\mathrm{TU}=(2,27-0,75) 10=15,2 \mathrm{t}$.

$$
\mathrm{Ft}=0,55-0,46 ; \frac{\mathrm{N}^{0}}{\mathrm{~N}}=0,85 ; \mathrm{Tu}=15,2 ; \mathrm{Kt}=0,25 \text {. }
$$

XV. Melancholia periodica. E. S... rro. Alter 40 Jahre. Betrat die Anstalt am 22. December 1902. Gewicht (2 Pud 231/2 Pfund) $42,53 \mathrm{~kg}$. Die Kranke leidet an periodischer Geistesstörung von melancholischem Cha-

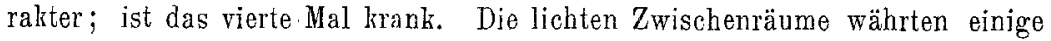
Jahre. Die Kranke hatte sich am 18. December drei Tage vor ihrer Ueberführung in die Anstalt unter den Zug geworfen, der aber noch rechtzeitig angehalten werden konnte. Während der Untersuohung der Oxydationsprocesse wurde der psychisohe Zustand der Patientin folgendermaassen beschrieben: Die Kranke weigert sich Speise zu sich zu nehmen und wird durch die Sonde genährt; hierzu kommt sie selbst, ohne sich zu widersetzen in das Esszimmer und fleht nur, dass man sie nicht füttern möge, da sie sterben will. Spricht leise; Gesichtsausdruck gespannt, finster. Puls 88-90', leicht erregbar; die inneren Organe innerhalb der Grenzen der Norm.

Gewicht: 5. Februar (2 Pud 291/2 Pfund) 44,49 kg; 19. Februar (2 Pud $23 \frac{1}{2}$ Pfund) 42,53 kg; 26. Februar (2 Pud 29 Pfund) $44,9 \mathrm{lg}$ und 5. März (2 Pud 32 Pfund) $45,8 \mathrm{~kg}$.

18. Februar. Tagesmenge des Harns $800 \mathrm{ccm}$; spec. Gew. 1017; $\mathrm{F}=0,001 ; \frac{\mathrm{N}^{0}}{\mathrm{~N}}=\frac{5,57}{8,94}=0,62$.

19. Februar. Tagesmenge des Harns 1450 ecm; spec. Gew. 1011; Spuren von Fhenol. Erhielt $1 \mathrm{~g}$ Benzol. $\mathrm{F}=0,35$.

20. Februar. Tagesmenge des Harns - $1320 \mathrm{ccm}$; spec. Gew. - 1014;

21. Februar. Tagesmenge des Harns $1950 \mathrm{ccm}$; spec. Gew. 1014; $\mathrm{F}=0,002 ; \mathrm{Ft}=0,35$.

22. Februar. Tagesmenge des Harns $1100 \mathrm{ccm}$; spec. Gew. 1012; $\frac{\mathrm{N}^{0}}{\mathrm{~N}}=0,64 ; \mathrm{F}=$ Spuren.

23. Februar. Erhielt $1 \mathrm{~g}$ Benzol.

24. Februar. Tagesmenge des Harns $1900 \mathrm{ccm}$; spec. Gew. 1013; $\mathrm{F}=0,369$. 
25. Februar. Tagesmenge des Harns $1300 \mathrm{ccm}: \mathrm{F}=0,04 ; \mathrm{Ft}=$ $0,409-0,004=0,405$.

26. Februar. Tagesmenge des Harns $1700 \mathrm{ecm;} \mathrm{spec.Gew.} \mathrm{1014;} \mathrm{Spuren}$ von Phenol.

$$
\mathrm{F}^{\mathrm{t}}=0,35-0,405 ; \frac{\mathrm{N}^{0}}{\mathrm{~N}}=0,62-0,64 \text {. }
$$

XVI. Psychosis periodica (Melanoholia). A. B...ko. Alter 47 Jahre. Wurde am 3. August 1904 zum dritten Mal in die Anstalt gebracht. Entstammt einer degenerirten Familie. Litt in der Jugend bäufjg an Nasenbluten. Die erste geistige Erkrankung trat im Alter von 14-15 Jahren auf; wurde damals nach ca. 6 Monaten gesund. Nach VerIauf von 5 Jahren trat ein neuer Anfall der Krankheit auf, der gleichfalls mit Genesung endete. Der dritte Anfall erfolgte nach 7 Jahren, der vierte nach 9 Jahren. Das letzte Mal war er im Jahre 1901-1902 krank gewesen, verliess die Anstalt am 4. Juni 1903 mit merklichen Anzeichen der Debilitas mentalis. Lebte bei seiner Mutter zu Hause und war seit dem Jahre 1891 nicht in Dienst. Die jetzige Erkrankung begann ziemlich plötzlich am 1. August 1904. Jeder einzelne Anfall besteht aus mehreren Anfällen eines schwer melancholischen stupiden Zustandes mit Temperaturerhöhung bis $37,6-37,8$ Grad, reichlichen Schweissergüssen, Unreinlichkeit etc. Solche Anfälle wechseln mit kurzdauernden Perioden der Erleichterung, während welcher die Temperatur sinkt, der Patient zu essen beginnt, spricht und zuweilen spazieren geht; dann tritt wieder der melancholisch-stupide Zustand ein, und so mehrere Male. Während des jetzigen Aufenthaltes in der Anstalt war der Zustand des Patienten folgender: liegt unbeweglich im Bett, die Nase geröthet, Mimik feblt, schwitzt, antwortet auf Fragen nur leise "schwer". Bei Fütterung mit dem Löffel isst er wenig, Puls $110-120^{\prime}, t^{0}=$ ca. 37,50. Stuhl nur nach Clysma; Malariaplasmodien wurden trotz zahlreicher Untersuchungen im Blut nicht gefunden. Seit Mitte August trat eine Besserung ein, und der Kranke fuhr sogar einmal allein zu seiner Mutter. in die Stadt. Am 13. September wiederholte sich der melancholisch-stupide Zustand; $t^{0}=37-37,4^{0}$; schwitzt, aber nicht so stark.

Vom 31. September an war die Temperatur normal, das Befinden bessert sich und der Patient verlässt bis zum 29. October das Bett; hiernach trat ein ziemlich schwerer Anfall auf; $t^{0}=37-37,9^{\circ}$. Im Anfang December war der Zustand besser, während der Mitte des Monats verschlimmerte sich das Befinden. Der ganze Cyclus von Anfällen endete erst vollständig im Frühling 1905, als wieder das gewöhnliche gute Befinden sich einstellte. Der Patient arbeitete, las; ging überall-viel spaziren, fuhr allein in die Stadt, war mittheilsam, guter Laune etc. Gewicht im August 1904: 5 Pud 4 Pfund - 5 Pud 3 Pfund 5 Pud 6 Pfund $(83 \mathrm{~kg}) ; 1$.- -8 . September: 5 Pud 12 Pfund -5 Pud 11 Pfund. 15. September: 4 Pud 39 Pfund (81 kg); 29. September: 5 Pad 4 Pfund (83kg). Im October: 5 Pad 4 Pfund -5 Pud 2 Pfund. 10. November: 4 Pud 39 Pfund $(81 \mathrm{~kg})$; im Januar 1905: 4 Pud 35 Pfund $(79,55 \mathrm{~kg})$. Im September und October 1905: 4 Pud 23 Pfund (75 kg); im September 1906: 3 Pud 33 Pfund (65 kg). 
21. September 1904. (Der schlcchte Zustand lässt nach.) Tagesmenge des Harns $1300 \mathrm{ccm}$; spec. Gew. 1023; Reaction alkalisch; Gefrierpunkt $=-2,19$. Zur Injection wurde ein Kaninchen von $1305 \mathrm{~g}$ verwendet; derTod erfolgte nach $11^{\prime} ; \mathrm{Tu}=(2,7-1,5) 13=16,9 \mathrm{t} ; \frac{\mathrm{N}^{0}}{\mathrm{~N}}=\frac{10,97}{15,01}=0,73$. Phenol im Harn nicht vorbanden; erhielt $1 \mathrm{~g}$ Benzol.

22. September. Tagesmenge des Harns $975 \mathrm{ccm}$; spec. Gew. 1024; Reaction alkalisch; viel Salze. $\mathbf{F}=0,34$.

23. September. Tagesmenge des Harns $900 \mathrm{ccm}$; spec. Gew. 1024. $\mathrm{F}=0,05 . \quad \mathrm{Ft}=0,39$.

24. September. Tagesmenge des Harns $1300 \mathrm{ccm}$, spec. Gew. 1023, Gefrierpunkt-2,02. Ein Kaninchen von $2005 \mathrm{~g}$ Gewicht verendete nach 14'. $\mathrm{Tu}=(2,2-1,16) 13=13,5 \mathrm{t}$.

1. October. ErhieIt $1 \mathrm{~g}$ Benzol. (Das Befinden des Patienten ist besser und $t^{0}$ normal.)

2. October. Tagesmenge des Harns $1300 \mathrm{ccm}$, spec. Gew. 1020. Reaction während der ganzen Zeit alkalisch. $F=0,34$.

3. October. Tagesmenge des Harns $1000 \mathrm{ccm}$, spec. Gew. 1022. $\mathrm{F}=0,003 . \mathrm{Ft}=0,343$.

5. December 1904. Temperatur normal, schwitzt stark, der physische Zustand besser, der psychische hat sich verhältnissmässig wenig gebessert. Tagesmenge des Harns $1200 \mathrm{ccm}$, spec. Gew. 1021, Gefrierpunkt - 1,94. Ein Kaninchen von $1525 \mathrm{~g}$ Gewicht verendete nach $9^{\prime}$. Ta $=(3,4-1,55) 12=$ 22,2 t. $\frac{\mathrm{N}^{0}}{\mathrm{~N}}=\frac{9,75}{12}=0,81$.

4. October 1905. Tagesmenge des Harns $2100 \mathrm{ccm}$, spec. Gew. 1013, Reaction neutral (amphoter), Gefrierpunkt $-1,16$. Ein Kaninchen von $1340 \mathrm{~g}$ Gewicht verendete nach $9^{\prime}$. $T u=(1,8-0,276) 21=33$ t. Erhielt $1 \mathrm{~g}$ Benzol.

5. October. Tagesmenge des Harns $2250 \mathrm{ccm}$, spec. Gew. 1012. $\mathrm{F}=0,31$.

6. October. Phenol im Harn nicht vorhanden.

23. September 1906. Tagesmenge des Harns $2850 \mathrm{ccm}$, spec. Gew. 1018, Reaction neutral, Gefrierpunkt - 1,64. Ein Kaninchen von $1980 \mathrm{~g}$ Gewicht verendete nach $32^{\prime} . T u=(1,43-0,533) 28,5=25,6 \mathrm{t}$.

$\begin{array}{llc}\text { Schwerer Zustand. } & \text { Besserung. } & \begin{array}{c}\text { Zufriedenstellendes } \\ \text { Befinden. }\end{array} \\ \mathrm{Ft}=0,39 & \mathrm{Ft}=0,346 & \mathrm{Ft}=0,31 \\ \mathrm{~N}^{0}=0,73 & \mathrm{~N}^{0}=0,81 & \mathrm{~N}^{0}=\text { nicht bestimmt } \\ \mathrm{Tu}=16,9-13,5 & \mathrm{Tu}=22,2 & \mathrm{Tu}=33,0-25,6 \\ \mathrm{Kt}=0,2-0,164 & \mathrm{Kt}=0,27 & \mathrm{Kt}=0,44-0,39 .\end{array}$

XVII. Paralysis progressiva. S. Ch ... ki. Betrat die Anstalt am 8. November 1901. Schauspicler. Alter 34 Jahre. Gewicht 3 Pud Archiv f. Psyehiatrie. Bd. 45. Heft 1. 
11 Pfund $(53,67 \mathrm{~kg})$. Entstammt einer degenerirten Familie, hat Lues, Die Krankheit entwickelte sich rasch im Laufe nur eines Monats vor der Ueberführung in die Anstalt; zu dieser Zeit wurde die charakteristische maniakalische Periode der progressiven Paralyse, mit Grössenwahnsinn beobachtet. Gegen Ende des Sommers and im Herbst 1902 zeigte sich bereits das Bild des ruhigen, paralytischen Schwachsinns. Gegen Ende des Jahres 1902 begannen die paralytischen Erscheinungen sich raschzuverstärken: Dysarthrie, Schwäche in den Füssen, Unreinlichkeit, zeitweilig convulsische Zuckungen, Verlust des Bewusstseins etc. Im Mai 1903 konnte der Kranke sich schon nicht mehr auf den Fï̈ssen haiten, starkes Zittern in allen Giliedern, zusammenhanglose, paralytische Sprache, Unreinlichkeit. Starb an 1. April 1904.

Gewicht im Mai 1903: 4 Pad 15 Pfund (55,2 kg) - 4 Pad 17 Pfund $(56,0 \mathrm{~kg})$.

15. Mai 1903. Harnmenge $1350 \mathrm{ccm}$, spec. Gew. 1014. $\mathrm{F}=0,006$. $\frac{\mathrm{N}^{0}}{\mathrm{~N}}=\frac{10,06}{12,76}=0,79$.

Erhielt 1 g Benzol.

16. Mai. Harnmenge $1000 \mathrm{ccm}$, spec. Gew. 1018. $\mathrm{F}=0,312$. $\frac{\mathrm{N}^{0}}{\mathrm{~N}}=\frac{8,35}{11,22}=0,74$.

17. Mai. Harnmenge $1150 \mathrm{ccm}$, spec. Gew. 1017. $\mathrm{F}=0,04 . \mathrm{Ft}=$ $0,352-0,012=0,34$.

24. Mai. Erhielt $0,99 \mathrm{~g}$ Benzol.

25. Mai. Harnmenge $1550 \mathrm{ccm}$, spec. Gew. 1017. $\mathrm{F}=0,221$.

26. Mai. Harnmenge $1300 \mathrm{ccm}$, spec. Gew. 1016. $\mathrm{F}=0,109$.

27. Mai. Harnmenge $1000 \mathrm{ccm}$, spec. Gew. 1021. $\mathrm{F}=0,05$.

$$
\mathrm{Ft}=0,221+0,109+0,05=0,38 \text {. }
$$

In Anbetracht dessen, dass im gegebenen Falle, trotz des sorgfältigen Sammelns des Harns, immer doch ein Theil desselben verloren ging, muss gefolgert werden, dass die Gesammtmenge des innerhalb 24 Stunden ausgeschiedenen Phenols grösser war, als die gefundene Menge.

$$
\mathrm{Tt}=0,34-0,38 . \quad \frac{\mathrm{N}^{0}}{\mathrm{~N}}=0,79-0,74 \text {. }
$$

XVIIl. Paralysis progressiva. P. S.... W. Wurde in die Anstalt am 7. März 1903 aufgenommen. Alter 39 Jahre, Gewicht 3 Pud 37 Pfund (64,2 kg). Erblichleit wird in Abrede gestellt; hatte vor 9Jahren Lues gehabt; trank viel, besonders während des letzten Jahres. War zwei Wochen vorher erkrankt. Bei seiner Ueberführung in die Anstalt waren die Lymphdrüsen vergrössert, die rechte Gesichtshälfte war schwächer jnnervirt als die linke. die rechte Pupille etwas mehr erweitert, primäre Reaction auf Licht and Accommodation normal, die secundäre Reaction etwas abgeschwächt, die Zunge nach links gerichtet, zittert, die Töne des Herzens etwas dumpf, der zweite an der Aorta accentuirt; die Gefässe sclerotisirt, Puls verlangsamt ca. 60'. Leber vergrössert, Darmkanal aufgedunsen, Knie- und vasomotorische Reflexe erhöht 
Hacken- und Unterleibsreflexe fehlen, Zittern in den Fingern. Leise, verlangsamte Sprache. Der Patient beschuldigt sich des Lasters, sagt, dass er ein Trinker ist, und dass er die hohe Institution, in der er gedient hat, entehrt babe, er verdiene Zwangsarbeit und nicht gute Pflege. Seinetwegen würden seine Kollegen und vielleicht auch ganz Petersburg zu Grunde gehen usw. Zeitweilig schweigt er und antwortet nicht auf Fragen. Während der Untersuchung der Oxydationsprocesse hatte der physische Zustand des Patienten sich merklich gebessert, er ass viel, der Darm functionirte gut. Die Wahnideen von Untergang und die Beängstigungen hatten sich im Laufe des März und der ersten Hälfte des April gelegt, gegen Mitte April verschwanden die melancholischen Wahnvorstellungen, es zeigte sich ein kritisches Verhalten zur Krankheit. Der Patient klagte über Schwächung der Aufmerksamkeit und des Denkvermögens und war etwas exaltirt. Geniesst vom Mai an vollkommene Freiheit, fährt in die Stadt. Blieb aus eigenem Antriebe bis zum 2. September in der Anstalt, die er ohne merkliche Anzeichen einer geistigen Störung verliess. In Betreff des liörperlichen Befindens wurde constatirt: Abschwächung der secundären Reaction auf Licht, Verstärkung der vasomotorischen und Sehnenreflexe. Hiernach war er noch einige Monate im Dienst, reichte aber darauf seinen Abschied ein. Ungefähr ein Jahr nachdem der Kranke die Anstalt verlassen hatte, begegnete ihm einer der Aerzte. Es zeigte sich hierbei eine gewisse Abstumpiung des Urtheils, das Zittern trat deutlich hervor, die Pupillen waren ungleichmässig erweitert, der Kranke sieht optimistisch auf Alles, lebt aber selbstständig.

Gewicht. 5. März 1903: 3 Pud 37 Pfund $(64,2 \mathrm{~kg})$. 19. März: 4 Pud 9 Pfund $(69,12 \mathrm{~kg})$. 2. April: 4 Pad 11 Pfund $(69,93 \mathrm{~kg})$. 15. April: 4 Pud 15 Pfund $(71,57 \mathrm{~kg})$. 30. April : 4 Pad 25 Pfund $(75,66 \mathrm{~kg})$. 7. Mai: 4 Pud 27 Pfund (76,48 kg). 21. Mai: 4 Pud 29 Pfund $(77,1 \mathrm{~kg})$. 28. Mai: 4 Pad $291 / 2$ Pfund. Verliess die Anstalt mit einem Gewicht ven 4 Pud 32 Pfund $(78,52 \mathrm{~kg})$.

23. März. 'T'agesmenge des Harns $2400 \mathrm{ccm}$; spec. Gew. 1014. Im Harn Spuren von Phenol; $\frac{\mathrm{N}^{0}}{\mathrm{~N}}=\frac{11,65}{15,36}=0,75$.

24. März. Tagesmenge des Harns $2650 \mathrm{ccm}$; spec. Gew. 1013; Spuren von Phenol; $\frac{\mathrm{N}^{0}}{\mathrm{~N}}=\frac{11,67}{14,10}=0,82$. Erhielt $1 \mathrm{~g}$ Benzol.

25. März. Tagesmenge des Harns $2850 \mathrm{com}$; spec. Gew. 1013; $\mathrm{F}=0,375$.

26. März. Tagesmenge des Harns $2100 \mathrm{com}$; spec. Gew. 1016; Spuren von Phenol.

29. März. Erhielt $1 \mathrm{~g}$ Benzol.

30. März. Tagesmenge des Harns $2600 \mathrm{ccm}$; spec. Gew. $1013 ; \mathrm{F}=0,356$.

31. März. Tagesmenge des Harns $2100 \mathrm{ccm}$; spec. Gew. 1016; Spuren ron Phenol. 
11. Mai. Tagesmenge des Harns $1650 \mathrm{ccm}$; spec. Gew. 1015; $\mathrm{F}=0$; $\frac{N^{0}}{\mathrm{~N}}=\frac{16,49}{18,31}=0,90$. Erhielt $1 \mathrm{~g}$ Benzol.

12. Mai. Tagesmenge des Harns $2150 \mathrm{ccm}$; spec. Gew. $1014 ; \mathrm{F}=0,3$.

13. Mai. Tagesmenge des Harns $1200 \mathrm{ccm}$; spcc. Gew. $1015 ; \mathrm{F}=0$.

14. Mai. Erhielt $1 \mathrm{~g} \mathrm{Benzol.}$

15. Mai. Tagesmenge des Harns $1700 \mathrm{ccm}$; spec. Gew. 1011; $\mathrm{F}=0,26$.

16. Mai. Tagesmenge des Harns $1600 \mathrm{ccm}$; spec. Gew. 1014; $\mathrm{F}=0$.

Während der Krankheit

$$
\begin{aligned}
& \mathrm{Ft}=0,376-0,356 \\
& \overline{\mathrm{NO}}=0,75-0,82
\end{aligned}
$$

Während der Besserung

$$
\mathrm{Ft}=0,3-0,26
$$$$
\frac{\mathrm{N}^{0}}{\mathrm{~N}}=0,90 \text {. }
$$

XIX. Paralysis progressiva. D. R...n. Wurde am 17. October 1903 in das Krankenhaus übergeführt. Alter 38 Jahre. Gewicht (3 Pud 28Pfd.), 60,53 lg. Entstammt einer degenerirten Familie; hatte Syphilis gebabt. Im. Mai 1903 begann die lustige Gemüthsstimmung, erhöbtes Selbstbefinden und Ueberschätznng der eigenen Person. Beim Eintritt in die Anstalt fehlten die Knie- and Unterleibsreflexe; Hacken- und vasomotorische Reflexe verstärkt. Die Zunge zittert und jst belegt. Zittern in den Händen. Die rechte Pupille mehr erweitert als die linke; Reaction anf Licht und Accommodation abgeschwächt. Sprache verschnellert. Handschrift leicht zitternd. Die Flerztöne dumpf. Puls $100^{\circ}$. Während der Untersuchung der Oxydationsprocesse traten von Seiten der Psyche die charakteristischen Erscheinungen der progressiven Paralyse auf; der Appetit vergrösserte sich, der Patient nahm an Gewicht zu, Verdanungsfunctionen gut. Am 12. April 1904 veriiess der Patient die Anstalt. mit gebessertem Befinden; wurde von seinen Verwandten als gesund betrachtet.

Gewicht im October (3 Pud $26 \mathrm{Pfd}$.) 59,7 kg; (3 Pud $28 \mathrm{Pfd}$.) 60,53 kg; boim Verlassen der Anstalt ( 3 Pud 28 Pfd.) 60,53 ligg.

20. October 1903. Tagesmenge des Harns $1650 \mathrm{ccm}$; spec. Gew. 1016; Phenol nicht vorhanden; erhielt $1 \mathrm{~g}$ Benzol; $\frac{\mathrm{N}^{0}}{\mathrm{~N}}=\frac{11,3}{12,7}=0,89$.

21. October. Tagesmenge des Harns $1530 \mathrm{ccm}$; spec. Gew. $1015 ; \mathrm{F}=0,42$; $\frac{\mathrm{N}^{0}}{\mathrm{~N}}=\frac{10,47}{12,22}=0,85$.

22. October. Tageswenge des Harns $1940 \mathrm{ccm}$; spec. Gew. 1015; Phenol nicht vorhanden; erhielt $1 \mathrm{~g}$ Benzol.

23. October. Tagesmenge des Harns $1850 \mathrm{ccm}$; spec. Gew. $1013 ; \mathrm{F}=0,38$.

24. October. Tagesmenge des Harns $1800 \mathrm{ccm}$; spec. Gew. 1015; Phenol nicht vorbanden.

Zum zweiten Male wurde D. R. . . n, am 30. August 1904 in die Anstalt gebracht, wog (3 Pud $27 \mathrm{Pfd}$.) $60 \mathrm{~kg}$. Seit dem Verlassen der Anstalt bis. zum Juni 1904 war er bei gutem Befinden gewesen, darnach begann aber die 
melancholische Periode der Krankheit. Beim Wiedereintritt waren beide Pupillen erweitert, die rechte aber stärker, als die linke; die Reactionen abgeschwächt; die Zunge trocken, zittert; Footor ex ore; Herztöne dumpf; Puls $104-110^{\prime} ; t^{0}=37-37,2^{\circ}$. Kniereflexe fehlen; Hacken-, Unterleibs und vasomotorische Reflexe verstärkt. Zittern in den Fingern; die Sprache leise, langsam und stockend. Seitens der Psyche eine scharf ausgedrückte Depression, mit Wahnideen von Ansteckung and Untergang. Zur Zeit der Untersuchung der Oxydationsprocesse rom 3. August bis 10. August liegt der Patient im Bett; der Gesichtsausdruck fast unbeweglioh und erschrocken; er versucht irgendwohin zu gehen, steht auf und fällt. Isst nur bei energischer Fütterung mit dem Löffel. Puls 100-110. Nach den entsprechenden Maassnahmen, hauptsächlich Abführungsmitteln und warmen Bädern bessert sich der Zustand ein wenig, so dass man beginnen lionnte den Patienten ins Freie zu bringen. Gewicht (3 Pud 27 Pfd.) $60 \mathrm{~kg}$.

3. August. Tagesmenge des Harns $600 \mathrm{~cm}$; spec. Gew. 1027; Gefrierpunkt $=-2,03 ;$ Spuren von Phenol $\frac{\mathrm{N}^{0}}{\mathrm{~N}}=\frac{5,3}{7,12}=0,74$. Ein Kaninchen von $1220 \mathrm{~g}$ Gewicht verendete im Laufe von $15^{\prime} ; \mathrm{Tu}=(1,3-0,8) 6=3,12$.

4. August. Im Harn Spuren von Phenol. Erhielt $1 \mathrm{~g}$ Benzol.

5. August. Tagesmenge des Harns $1060 \mathrm{ccm}$; spec. Gew. 1017: $\mathrm{F}=0,519$; $\frac{\mathrm{N}^{0}}{\mathrm{~N}}=\frac{9,18}{10,9}=0,83$.

6. August. Im Harn Spuren yon Phenol.

9. Angust. Erhielt ron Neuem $1 \mathrm{~g}$ Benzol.

Die gesammte Harnmenge vom 10. und 11. August - $1100 \mathrm{ccm}$; spec. Gew. $1022 ; \mathrm{F}=0.47$.

12. August. Tagesmenge des Harns $600 \mathrm{ccm}$; spe. Gow. 1022; Spuren von Phenol; Gefrierpunkt $=-1,8$. Ein Kaninchen von $1320 \mathrm{~g}$ Gewicht verendete nach $20^{\circ}$. Ta $=(1,26-0,618) 6=3,25 \mathrm{t}$.

Im April 1905 trat in dem äusserst schIimmen Befinden des Patienten wieder eine Besserung ein; der Kranke wurde reinlich, begann selbst zu essen, ging viel im Freien spazieren; spielte Karten, machte einer der Gehülfinnen de: Aufseherin den Hof; der Aufenthalt in der Abtheilung für Schwache wurde ihm unangenehm, so dass er in die Abtheilung für ruhige und bewusste Kranke übergeführt wurde. Seit dem August genoss er im Bereich des Krankenhauses volle Freiheit, las, spielte Billard, interessirte sich für Politik. Die Erscheinungen des Schwachsinns waren deutlich ausgedrückt. In der folgenden Zeit überstand der Kranke noch eine Erregung in Gestalt einer Reiheapoplectiformer Anfälle, berahigte sich aber bald von Neaem und befand sich im Herbst 1906 wieder in der ruhigen Abtheilung.

Gewicht im October 1905 - 4 Pud $(65 \mathrm{~kg})$.

2. September 1905. Tagesmenge des Harns $1600 \mathrm{com}$; spec. Gew. 1014;

Gefrierpunkt $=-1,23$. Fin Kaninchen von $1200 \mathrm{~g}$ Gewicht verendete nach $19 . \mathrm{Tu}=(1,5-0,447) 16=16,85$. 
4. October. Tagesmenge des Harns $-1900 \mathrm{ccm}$; spec. Gew. 1012; Gefrierpunkt $=-1,19$. Ein Kaninchen von $970 \mathrm{~g}$ Gewicht verendete nach 8 '. $\mathrm{Tu}=(1,17-0,22) 19=18,0$. Erhielt $1 \mathrm{~g}$ Benzol.

5. October. Tagesmenge des Harns $1950 \mathrm{ccm} ; \mathrm{F}=0,37$.

$$
\begin{array}{ccc}
\text { Maniakalische Periode } & \text { Melancholische Periode } & \text { Lichte Periode } \\
\mathrm{Tt}=0,42-0,38 & \mathrm{Ft}=0,519-0,47 & \mathrm{Ft}=0,37 \\
\frac{\mathrm{N}^{0}}{\mathrm{~N}}=0,89-0,85 & \frac{\mathrm{N}^{0}}{\mathrm{~N}}=0,74-0,83 & \\
& \mathrm{Tu}=3,12-3,25 & \mathrm{Tu}=16,85-18,0 \\
& \mathrm{Kt}=0,052-0,054 & \mathrm{Kt}=0,26-0,27
\end{array}
$$

XX. Psychosis neurasthenica (degenerativa). E. K...li. Betrat die Anstalt am 16. April 1904. Alter 39 Jahre; entstammt einer degenerirten Familie. Die Kranke war 13 Jahre verheirathet; zwei der Kinder waren gestorben, vier sind am Leben; die Patientin war immer argwöhnisch und furchtsam gewesen und liebte sehr, sich behandeln zu lassen. Nach ihrer letzten Niederkunft mit einem todten Kinde, im Januar 1902, stellten sich Anfälle der äussersten Schwäche ein; Argwohn und Todesfurcht verstärkten sich noch mehr. Seit dem 15. Mai 1905 wurde sie in einer der provinzialen psychiatrischen Kliniken unter der Diagnose Psychosis hysterica behandelt; darauf war sie in der Nervenabtheilung eines Petersburger Krankenhauses, wo zuerst die Aufmerksamkeit auf die ungenügende Function der Gl. thyreoideae gelenkt. wurde. Von der physischen Seite wurde während der Untersuchung der Oxydationsprocesse beobachtet: Ernährung ziemlich befriedigend. Die Haut und die sichtbaren Schleimhäute blass. Haut weich; Pupillen gleichmässig; Reaction auf Licht schwach; Kniereflexe normal, Hacken- und vasomotorische Reflexe verstärkt, Schluckreflex erhalten. Schmerzende Stellen über den beiden Brustdrüsen und im Gebiet des rechten Ovariums. Die Gl. thyreoideae lassen sich nicht durchfühlen. Herztöne gedämpft; Puls weich, leicht zusammendrückbar; sehr häufig nur 60 pro Min., aber leicht erregbar und verschnellert sich dann. Nach der Eingabe von Thyreoidin wurde der Puls entschieden besser, war nicht unter 76-80' und wurde voller. Eine Besserung des psychischen Zustandes habe ich nicht beobachtet und auch die Kranke selbst fühlte sich nicht besser. Arzeneien weigerte sich die Patientin einzunehmen und fürchtete sich besonders vor Thyreoidin. Obgleich die Kranke gesund zu werden wünscht, erfüllt sie die Rathschläge der Aerzte nicht, ist argwöhnisch, eigensinnig, äussert eine Menge der verschiedenartigsten Befürchtungen für ihr Herz und ihr Leben. Schwächung der Kritik und Sinken der Ethik. Verliess die Anstalt am 20. Juli 1904.

25. April 1904. Phenol im Harn nicht vorhanden. Nahm $1 \mathrm{~g}$ Benzol ein.

26. April. Tagesmenge des Harns $1050 \mathrm{ccm}$; spec.Gew.1020; $\mathrm{F}=0,09$; $\frac{\mathrm{N}^{0}}{\mathrm{~N}}=\frac{11,23}{12,46}=0,90$.

27. April. Tagesmenge des Harns $950 \mathrm{ccm}$; spec. Gew. $1021 ; \mathrm{F}=0,03$. $\mathrm{Ft}=0,12$. 
Vom 27.-30. April täglich ein Mal 0,2 Thyreoidin.

29. April. Erhielt $1 \mathrm{~g}$ Benzol.

30. April. Tagesmenge des Harns $1400 \mathrm{~cm}$; spec. Gew. 1017; $\mathrm{F}=0,195$; $\frac{\mathrm{N}^{0}}{\mathrm{~N}}=\frac{12,52}{14,0}=0,90$.

1. Mai. Tagesmenge des Harns $1300 \mathrm{ccm} ; \mathrm{F}=0,015$. $\mathrm{Ft}=0,210$.

Vom 10.-20. Mai erhielt die Kranke täglich zwei Mal 0,2 Thyreoidin.

18. Mai. Erhielt $1 \mathrm{~g}$ Benzol.

19. Mai. Tagesmenge des Harns $1700 \mathrm{ccm}$; spec. Gew. 1014; $F=0,203$; $\frac{\mathrm{N}^{0}}{\mathrm{~N}}=\frac{20,93}{21,24}=0,94$.

20. Mai. Tagesmenge des Harns $1750 \mathrm{ccm}$; spec. Gew. 1014; $\mathrm{F}=0,12$. $\mathrm{Ft}=0,323$.

\begin{tabular}{|c|c|c|}
\hline Ohne Thyreoidin & Thyreoidin 0,2 & Thyreoidin $0,3 \times 2$ \\
\hline $\mathrm{Ft}=0,12$ & $F t=0,21$ & $\mathrm{Ft}=3,23$ \\
\hline$\frac{\mathrm{N}^{0}}{\mathrm{~N}}=0,90$ & $\frac{\mathrm{N}^{0}}{\mathrm{~N}}=0,90$ & $\frac{\mathrm{N}^{0}}{\mathrm{~N}}=0,94$. \\
\hline
\end{tabular}

XXl. Morbus Basedowii. M. R ... skaja. Wurde vom Autor ambulatorisch behandelt; die Kranke war 46 Jahre alt; Witiwe; ziemlich boch gewachsen; Ernährung geschwächt; das (subcutane Gewebe) Unterhautgewebe äusserst schlecht entwickelt; Gewicht (3 Pud $30 \mathrm{Pfd}$.) $61,35 \mathrm{~kg}$. Entstammt einer Familie, die zu Nervenkrankheiten geneigt ist. Kopfschmerzen stellten sich im Alter der Geschlechtsreife ein. Eine merkliche Vergrösserung des Kropfes begann im 30. Lebensjahre. Zur Zeit sind beide Hälften der Schilddrüse beträchtich vergrössert; die Augenäpfel treten wenig hervor; Puls 110-120', erreicht aber bei der geringsten Erregung 140-150' und noch mehr. Fine auffällige Verstärkung der vasomotorischen und Sebnenreflexe. Die Kranke ist leicht reizbar, schläft schlecht, leidet beständig an Kopfschmerzen und Schmerzen im Gebiet des Herzens. Liess sich viel von verschiedenen Aerzten behandeln. Im Jahre 1902 wurden ihr im Laufe eines Monats subcutane Einspritzungen von Spermin (Poehl) gemacht, und während dieser Zeit fühlte die Kranke sich besser. Vom Ende April 1904 nahm sie im Lâufe von zwei Monaten tägich zwei Mal 20-35 Tropfen Essentiae Spermini Poehl ein. Fühlte sich hiernach besser, die Kopfschmerzen verschwanden fast, und sie war weniger nervös. Die Besserung hielt noch zwei Monate nach Abbruch der Behandlung mit Spermin an.

14. April 1904. Im Harn ist Phenol nicht vorhanden, Erbielt $1 \mathrm{~g}$ Benzol.

15. April. Tagesmenge des Harns $2000 \mathrm{ccm}$; spec. Gew. 1013; $\mathrm{F}=0,504$; $\frac{\mathrm{N}^{0}}{\mathrm{~N}^{-}}=\frac{10,05}{12,18} 0,83$.

16. A pril. Tagesmenge des Harns $1900 \mathrm{ccm}$; spec. Gew. 1013; $\mathrm{F}=0$. Erhielt $1 \mathrm{~g}$ Benzol. 
17. April. Tagesmenge des Harns $2600 \mathrm{ccm}$; spec. Gew. $1009 ; \mathrm{F}=0,38$. $\frac{\mathrm{N}^{0}}{\mathrm{~N}}=\frac{9,95}{12,1}=0,82$.

18. April. Tagesmenge des Harns $2000 \mathrm{ccm}$; spec. Gew. $1011 ; \mathrm{F}=0,04$. $\mathrm{Ft}=0,42$.

Vom 18. April an nahm die Kranke täglich zwei Mal 25 Tropfen Essentiae Spermini Poehl innerlich ein.

21. April. Erhielt $1 \mathrm{~g}$ Benzol.

22. April. Tagesmenge des Harns $2500 \mathrm{com}$; spec. Gew. 1011; $\mathrm{F}=0,264$

23. April. Tagesmenge des Harns $2150 \mathrm{~cm} ; \mathrm{F}=0$.

Vom 26. April an erhielt die Kranke täglich zwei Mal 35 Tropfen Essentiae Spermini Poehl innerlich.

1. Mai. Erhielt $1 \mathrm{~g}$ Benzol.

2. Mai. Tagesmenge des Harns $2050 \mathrm{ccm}$; spec. Gew. 1012; $\mathrm{F}=0,206$; $\frac{\mathrm{N}^{0}}{\mathrm{~N}}=\frac{11,12}{12,48}=0,89$.

3. Mai. Tagesmenge des Harns $1950 \mathrm{ccm}$; spec. Gew. 1014; $\mathbf{F}=0$.

Ohne Spermin Spermin 25 Tropfen $\times 2$ Spermin 35 Tropfen $\times 2$. $\mathrm{Ft}=0,504-0,42 \quad \mathrm{Ft}==264$ $\frac{\mathrm{N}^{0}}{\mathrm{~N}}=0,83-0,82$ $\mathrm{Ft}=0,206$ $\frac{\mathrm{N} 0}{\mathrm{~N}}=0,89$.

Die Untersuchung der Oxydationsprocesse im Organismus von vier gesunden Menschen von verschiedenem Geschlecht, Beruf und Ernährung zeigt, dass aus $1 \mathrm{~g}$ per os in den Organismus eingeführten Benzol $0,3-0,314-0,32-0,34$ und $0,36 \mathrm{~g}$ Phenol gebildet wurden; im Durchschnitt also 0,328 d. h. rund ca. 0,33 g Phenol. Der Coefficient der Oxydationsenergie nach Prof. Poehl betrug bei denselben gesunden Nenschen meinen Beobachtungen nach $=0,85-0,88-0,89-0,93$ oder im Durchschnitt 0,88 . Die von mir gefundenen Zablen sind etwas niedriger als die von Prof. Poehl angegebenen. Die Anzahl der Toxieen d. h. der Giftigkeitseinheiten (eine Einbeit tödet ein $\mathrm{kg}$ Thier) fand ich in der Tagesmenge des Harns gesunder Menschen gleich 5,0 - 5,138,12 - 9,39 oder im Mittel 6,9 Toxieen. Der urotoxische Coefficient d. h. die Zahl, die durch Division der Anzahl Toxieen, die mit dem Harn innerhalb 24 Stunden ausgeschieden wird, durch die Zahl der Kilogramme des Gewichtes, betrug 0,072 - 0,081 - 0,117 - 0,146 oder im Mittel $=0,101$. Dieser Coefficient ist um mehr als 4 Mal kleiner als der von Bouchard erbaltene. Ich glaube aber, dass meine Resultate näher der Wahrheit sind, da Bouchard bei seinen Untersuchungen über die Giftigkeit des Harns nicht die äusserst wichtige Bedeutung des osmotischen Druckes des in das Blut eingeführten Harns 
in Betracht zog und natürlich nicht die, erst in Folge ausgearbeiteten, Vorsichtsmaassregeln einhalten konnte, bei meinen Versuchen dagegen fast alle zur Zeit bekannten Correctionen eingeführt wurden.

Wenn wir die Resultate der Untersuchungen der Oxydationsprocesse (eigentlich der Oxydation von Benzol) bei Gesunden und Geisteskranken vergleichen, fällt ein beträchtlicher Unterschied sofort in die Augen fast bei allen Kranken finden wir eine Störung der Oxydationsprocesse. Natürlich darf nicht ausser Acht gelassen werden, dass ich zu den Untersuchungen gerade solche Kranke wähIte, bei denen, auf Grund von verschiedenen Thatsachen, im Voraus eine Veränderung der Oxydationsprocesse erwartet werden musste. So wird die Krankheitsgeschichte zweier Patienten, an denen dieses nicht im genügenden Grade aufgeklärt werden kounte, nicht angeführt. Eine Verminderung der Oxydation wurde in den Fällen V (Paranoia chronica et tubercul. pneumon.), VI (Melancholia hysterica), VII (Melancholia periodica senilis), theilweise auch VIII (Melancholia senilis), IX (Psychosis eircularis - Melancholia) und endlich XX (Psychosis neurasthenica) an einer Kranken, die an mangelhafter Function der Schilddrüse litt, beobachtet. Des Falles V erwäbne ich nur hier, da es schwer zu unterscheiden ist, wodurch hier die Abschwächung der Oxydationsvorgänge bedingt wird, durch den Process, der die Geistesstörung verursacht, oder durch die specifische, organische (durch tuberculösen Process) Beschädigung der Lungen. Nach den klinischen Formen waren die geistigen Erkrankungen, bei denen eine Abschwächung der Oxydationsprocesse beobachtet wurde, immer melancholische Zustände mit verschiedenen klinischen Eigenthümlichkeiten. Drei von den Patienten standen bereits in höherem Alter. Interessant ist der Fall VII, bei welchem während der Krankheit die Oxydation des Benzols abnahm und nach der Geuesung des Kranken deutlich zunahm. In Fall VIII erfolgte die Verstärlkung der Oxydationsprocesse unter dem Einfluss subcutaner Injectionen vou physiologischer Kochsalzlösung, wobei die Verstärkung der Oxydationsprocesse von einer gewissen Besserung des Zustandes der Kranken und einer Erböhung des Coefficienten der Oxydationsenergie begleitet war; ebenso muss in Fall IX auf die starke Erhöhung der Oxydation des Benzols bei einer gewissen Besserung des Befindens der Patientin hingewiesen werden; die Beobacbtungen über diese Kranke sind aber noch nicht abgeschlossen. Das bei weitem grösste Interesse stellt Fall XX dar. Hier gab das klinische Bild der Krankheit Veranlassung, eine Atrophie der Schilddrüse voranszusetzen.

Die Untersuchung der Oxydationsprocesse nach der Methode von Nencki und Sieber zeigte bei dieser Kranken eine auffallende Ab- 
schwächung dieser Processe. Auch an Kaninchen konnte ich nach Fntfernung eines Theils oder der ganzen Glandula thyreoïdea eine Verminderung der Oxydation des Benzols beobachten. Somit bestätigten die Untersuchung der Oxydationsprocesse im vorliegenden Falle vollkommen die klinischen Voraussetzungen. Von einer Parallele zwischen Fall XX und den anderen Kranken, bei denen gleichfalls eine Verminderung der Oxydation des Benzols beobachtet wurde, zu sprechen, glaube ich aber nicht berechtigt zu sein, da ich hierzu nicht über genügend erforsehtes Thatsachenmaterial verfüge.

Die Verordnung von Thyreoïdin hatte in Fall XX nicht eine Besserung im psychischen Zustande der Kranken zur Folge, was durch viele Ursachen bedingt sein kann; vor Allem ist es bekannt, dass das käufliche Thyreoïdin (Glandula thyreoida sicca) niemals die Functionen der Schilddrüse völlig ersetzen kann; weiter waren, möglicher Weise, bei der Kranken ausser der Störung der Functionen der Glandula thyreoidea auch die Functionen anderer wichtiger Organe in Mitleidenschaft gezogen und endlich war eine regelrechte Behandlung der Kranken mit Thyreoidin nicht durchgeführt worden, in Folge der Weigerung ibrerseits, dieses Mittel einzunehmen. Es muss noch darauf hingewiesen werden, dass sich der Coefficient Poehl's bei dieser Kranken fast gar nicht verändert hatte. Unter dem Einfluss von Thyreoidin vergrösserte er sich auch ein wenig.

Im vollkommenen Gegensatz zu Fall XX steht Fall XXI. Auf Grund der klinischen Beobachtungen musste hier eine Hypersecretion der Glandula thyreoidea erwartet werden. Die Untersuchung der Oxydationsprocesse bestätigte dieses demonstrativ. Aus $1 \mathrm{~g}$ Benzol, das in den Organismus eingeführt wurde, bildeten sich $0,504 \mathrm{~g}$ Phenol. Auf Grund meiner experimentellen Untersuchungen war mir bekannt, dass Spermin die Oxydation des Benzols in Organismus herabsetzt. Als der Patientin nun Spermin verordnet wurde, sank die Oxydation des Benzols in ihrem Organismus fast um die Hälfte. Der Oxydationscoefficient Poehl's war bei dieser Kranken erniedrigt, unter dem Einfluss von Spermin stieg er jedoch von $0,82-0,89$. Die Veränderungen der Oxydationsprocesse durch die Wirkung des Spermins waren von einer Besserung im Zustande der Kranken begleitet.

Eine Zunahme der Oxydationsprocesse beobachtete ich bei zwei Katatonikern (Fall X und XI), zwei Paralytikern (Fall XII und XIII), und einem an degenerativer Psychose leidenden Kranken (XIV). Die letzten drei Fälle kamen der Krankheitsform der Dementia paranoides (Kraepelin) am nächsten.

Weiter wurde eine Zunahme der Oxydation des Benzols in zwei 
Fällen von periodischer Psychose mit melancholischen Eigentbümlichkeiten ( $\mathrm{XV}$ und $\mathrm{XVI}$ ) und ${ }^{i}$ endlich in drei Fällen von progressiver Paralyse beobachtet (XVII, XVIII und XIX). Bei dem Katatoniker $\mathrm{K}$... ge (Fall XI) trat während des schweren Krankheitszustandes eine Verstärkung der Oxydationsprocesse herror, die mit der Besserung des physischen und psychischen Zustandes des Kranken wieder zur Norm zurückkehrte. Eine besondere Beachtung verdient die auffällige Verstärkung der Oxydation des Benzols im Organismus von drei an Dementia paranoides kranken Personen; diese Verstärkung betrug bis $0,595 \mathrm{~g}$, was bei keinem der anderen Kranken gefunden wurde. Diese Thatsache ist in der Hinsicht interessant, dass sie einigen klinischen Beobachtungen, die auf einen Zusammenhang der Dementia paranoides mit Katatonia und Störungen der Function der Gland. thyreoidea binweisen, entspricht. Eine Zunahme der Oxydation des Benzols bei zwei periodischen Melancholikern ( $\mathrm{XV}$ und $\mathrm{XVI}$ ) weist darauf hin, dass die Störungen der Processe der Metamorphose bei diesen Kranken nicht die gleichen waren, wie bei den anderen periodischen Kranken, bei denen eine Abschwächung der Oxydationsprocesse beobachtet wurde. Augenscheinlich besteht zwischen den einzelnen Kranken ein tiefer Unterschied, ungeachtet der gemeinsamen Symptome des klinischen Bildes. Die Verstärkung der Oxydationsprocesse bei einigen Periodikern lässt die Frage aufkommen, in welchem Verhältniss diese periodischen Psychosen zu der Katatonie und den ihr verwandten Krankheiten stehen. Ich hatte schon einmal Gelegenheit gehabt, vor fünf Jahren in einer meiner Arbeiten ${ }^{1}$ ) die Aufmerksamkeit auf diese Frage zu lenken. Der Fall XVI ist in der Beziehung interessant, dass er zeigt, wie der Process der Oxydation des Benzols sich verändert, wenn der Zustand des an periodischer Psychose leidenden Kranken sich zu bessern anfängt und die lichte Periode beginnt. Ebenso ist ein Ansteigen der Oxydationsprocesse in den drei von mir untersuchten Fällen von progressiver Paralyse nicht zu verkennen. Freilich sind die erhaltenen Phenolmengen bei den Paralytikern, besonders in zwei Fällen (XVII und XVIIJ), nicht hoch; es muss jedoch in Betracht gezogen werden, dass bei dem Kranken S. Ch ... ki während der schweren Krankheitsperiode, ungeachtet der Sorgfalt beim Sammeln des Harns, immer doch ein Theil verloren ging; im Falle XVIII erwies es sich aber, dass nach Eintrits der Besserung die Processe der Oxydation des Benzols im Organismus abnahmen. Der Fall XIX zeigt, dass die Erhöhung der

1) A. J. Juschtschenko. Zur Frage über die Heilbarkeit der periodischen Psychosen. Russischer Medicinischer Anzeiger 1901. 
Oxydationsprocesse bei progressiver Paralyse gerade im Wesen dieser Kranken liegt, weshalb sie sich auch sowohl bei melancholischem, als auch bei maniakalischem Zustande der Kranken äussert; ja sogar bei melancholischem Zustande die Oxydationsprocesse energischer verlaufen als bei maniakalischem Zustande; mit fortschreitender Besserung und während der normalen Perioden sinken die Oxydationsprocesse und nähern sich der Norm.

Der Coefficient der Oxydationsenergie (nach Prof. Poehl) war bei der Mehrzahl der von mir uncersuchten Kranken mehr oder weniger vermindert. Am stärksten war diese Abnahme in den Fällen VI, VII, XVI, XVII und XVIII, wo der Coefficient, statt der von mir gefundenen normalen Grösse - 0,88, bis auf $0,83-0,73$ und 0,60 sank, wobei die Verminderung des Coefficienten in keiner directen Abhängigkeit von der Zunahme oder Abnahme der Oxydation des Benzols im Organismus stand. Jeder Veränderung der Oxydationsprocesse im Organismus der Geisteskranken entsprach ein mehr oder weniger starkes Sinken des Coefficienten der Energie der Oxydationsprocesse Prof. Poehl's. Mit der Besserung des Befindens der Kranken stieg dieser Coefficient immer an und näherte sich der Norm (VII, XVI, XVIII und XXI). Ohne somit die Bedeutung dieses Coefficienten der Oxydationsenergie zu leugnen, müssen wir jedoch zugeben, dass er die Frage von der chemischen Metamorphose im Organismus der Geisteskranken aur einseitig beleuchtet, während uns die Methode von Nencki und Sieber gestattet, das Wesen des Processes von einer breiteren Grundlage aus zu verfolgen.

Die Toxicität des Harns wurde in sechs Fällen geprüft. Im Falle V wurde keine besondere Veränderung der Toxicität des Harns constatirt; in einem Falle von Dementia paranoides (XIV) war die Toxicität des Harns erhöht, der urotoxische Coefficient $=0,25$, während er im Mittel bei gesunden Menschen 0,101 betrug. Von besonderem Interesse sind jedoch die vier anderen Fälle, wo die Toxicität des Harns sowobl. während des schweren Krankheitszustandes, wie auch während der freien Intervalle untersucht wurde. Im Falle IX bei einer circulären Kranken entsprach die Toxicität des Harns während des schweren melancholischen Zustandes 12,62 $\mathrm{T}$ und der urotoxische Coefficient - 0,22, bei der Besserung sank die Toxicitat bis auf 3,28-3,64 Toxieen in 24 Stunden und der urotoxische Coefficient bis auf $0,061-0,07$. Weiter wurde bei ihr wieder eine Erhöhung der Toxicität beobachtet; die Beobachtungen über diese Kranke wurden nicht zum Abschluss gebracht, es ist möglich, dass bei ihr eine neue Erregung beginnt.

Im Falle XI, bei einem Katatoniker, betrug der urotoxische 
Coefficient während des schweren Zustandes $0,2-0,204$ und sank bei der Besserung bis auf 0,07 und 0,055 , also um mehr als drei Mal, wobei von besonderem Interesse ist, dass die T'oxicität geringer wurde als die mittlere Toxicität des Harns gesunder Menschen. Diese Verminderung der Toxicität des Harns war von einer Veränderung der Oxydation des Benzols und der Coefficienten der Oxydationsenergie von Prof. Poehl begleitet. Augenscheinlich hatte sich im Organismus des Kranken entweder ein neuer Factor gebildet, oder es war ein anderer Factor ausgeschieden, wodurch die Metamorphose in den Geweben verändert wurde und eine Verminderung der Bildung oder eine Verstärkung der Zerstörung der Toxine im Organismus stattfand. Im vollkommenen Gegensatz zu den vorhergehenden Untersuchungen steht der Fall XIX, wo während der schweren melancholischen Periode der progressiven Paralyse die Toxicität des Harns stark vermindert war, der urotoxische Coefficient betrug - 0,054-0,052; als aber ein freies Intervall eintrat, stieg die Toxicität bis zu einer Höhe, die die Norm um das Doppelte übertraf, der urotoxische Coefficient $=0,26-0,27$. Diese Beobachtung zeigt, dass eine Verminderung der Toxicität des Harns während der schweren Kraukheitsperioden des Patienten keineswegs darauf hinweist, dass auch im Organismus der Toxingehalt des Blutes ein geringer ist. Im gegebenen Falle deutet das schwere klinische Bild der Krankheit augenscheinlich auf Autointoxication hin, jedoch werden die Toxine aus irgendwelchen Gründen im Organismus zurückgehalten und können nicht in den Harn übergehen; als nun aber die Ausscheidung der Toxine begann, verminderte sich ihre Menge im Blut, und der Organismus fing an, sich zu erbolen. Die Toxicität des Harns überstieg auch während der freien Intervalle die Norm, da die Oxydationsprocesse, wenngleich sie auch abgenommen hatten, immer noch die Norm übertrafen.

Etwas anders war die Sachlage in Fall XVI. Bei diesem Patienten war die Toxicität des Harns während der schweren Krankheitsperiode eile ziemlich hohe; der urotoxische Coefficient $=0,2-0,104$. Man konnte somit erwarten, dass mit dem Nachlassen der Krankheit auch die Toxicirăt sinken würde; die weiteren Untersuchungen zeigten hingegen, dass mit der Besserung des psychischen und physischen Zustandes des Organismus die Toxicität weiter zunahm und während des freien Intervalles, wo der Patient fast die Lebensweise eines gesunden Menschen führte, einen äusserst hohen Grad erreichte, so dass der urotoxische Coefficient $=0,44-0,39$ (!) war. Augenscheinlich war auch hier während der Krankheit die Toxicität des Blutes eine hohe gewesen, der Organismus war jedoch aus irgend welchen Gründen im Stande gewesen, sich der Giftstoffe zu entledigen; in der freien Periode wird nun, unge- 


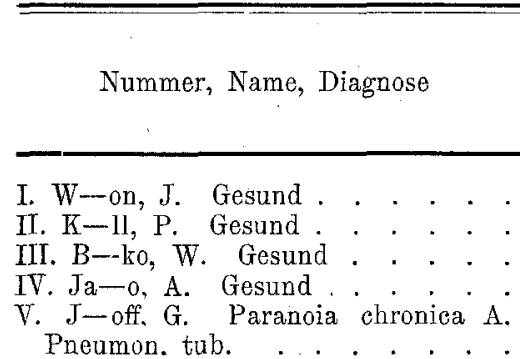

VI, P-off, M: Melancholia hystérica VII. J-off, J. Melancholia period. Erholt

VIII. B-wa, E. Melancholia senilis . Nach subcutaner Einverleibung der physiolog. Cl-Na-Lösung . . . .

IX. K-wa, A. Psychosis; cireal. Stad. Melancholia .

Besserung

X. N-eff, W. Katatonia.

XI. K-ge, W. Katatonia

Besserung

XII. S-ne, N. Dementia paranoides (Paranoia ohronica)

XIII. F-ff, B. Dementia paranoides (Paranoia chronica)

XIV. S-off, A. Dementia paranoides (Psychosis degenerat.)

XV. S-ro, E. Melancholia Psychosis periodica .

XVI. B-ko, A. Melancholia (Psychosis periodica)

Besserung

Lichte Periode.

XVII. H-ki, S. Paralysis progressiva

XVIII. S-oft, P. Paralysis progressiva Lichte Periode.

XIX. P-nn, D. Paralysis progressiva Stad. moniak.

Stad. melanchol.

Cichte Periode.

XX. K-li; E. Psychosis neurasthenica degenerativa

Tyreoidea 0,2 .

Tyreoidea $0,3+2$

XIX. N-aja, M. Morbus Basedowii Spermin. $25 \times 2$ Spermin. $35 \times 2$

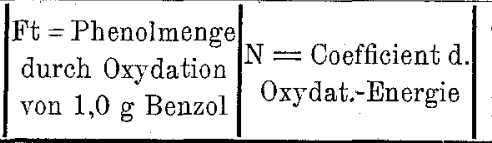

$\mathrm{Tu}=$ Zahl der Toxie 24 Stunden $\mathrm{Kt}=$ urotoxische $\mathrm{Cot}$

$\mathrm{Tu}=9,39 ; \mathrm{Kt}=0,1$

$0,34-0,36$

Nicht bestimmt

0,31

$0,208-0,23$

$0,307-0,2$

0,214

0,338

0,3

0,365

0,26

0,49

$0,376-0,367$

$0,466-0,4.07$

0,31

$0,473-0,595$

$0,338-0,427$

$0,55-0.46$

$0,35-0,407$

0,39

0,36

0,31

$0,34-0,38$

$0,367-0,356$

$0,3-0,26$

$0,42-0,38$

$0,510-0,49$

0,37

0,12

0,21

0,323

$0,504-0,42$

0,264

0,206
0,85

$0,88-0,89$

Nicht bestimmt 0,93

0,78

0.60

0,87

0,88

$0,66-0,71-0,72$

$0,80-0,85$

Nicht bestimmt

$0,86-0,75$

$0,89-0,82$

Nicht bestimmt

$0,79-0,81$

$0,83-0,84$

0,82

$0,62-0,64$

0,73

0,81

Nicht bestimmt

$$
0,79-0,74
$$

$0,75-0,82$

0,90

$0,89-0,85$

$0,74-0,85$

Nicht bestimmt

0,90

0,90

0,94

$0,83-0,82$

Nicht bestimmt 0,89
Nicht bestimmt

$\mathrm{Tu}=5,13 ; \mathrm{K} t=0$

$\mathrm{Tu}=5,0-8,12$;

$\mathrm{Kt}=0,072-0,66$

$\mathrm{Tu}=5,9-6,6 ;$

$\mathrm{Kt}=0,115-0,12$

Nicht bestimmt

$\mathrm{Tu}=12,62 ; \mathrm{Kt}=0$

$\mathrm{Tu}=3,28-3,65-7$

$\mathrm{Kt}=0,063-0,07-\mathrm{t}$

Nicht bestimmt

$\mathrm{T} \mathrm{u}=8,8-8,93$;

$\mathrm{Kt}=0,2-0,204$

$\mathrm{Tu}=2,37-1,87$

$\mathrm{Kt}=0,07-0,05 \mathrm{t}$

Nicht bestimmt

$\mathrm{T} u=15,2: \mathrm{Kt}=0$,

Nicht bestimmit

$\mathrm{Tu}=16,9-13,5 ;$

$\mathrm{Rt}=0,2-0,169$

$\mathrm{Tu}=22,2 ; \mathrm{Kt}=0$ ，

$\mathrm{Tu}=33,0-25,0$ :

$K t=0,44-0,39$

Nicht bestimmt

$\mathrm{Tu}=3,12-3,25:$

$\mathrm{Kt}=0,052-0,05$.

$\mathrm{Tu}=16,85-18,0$

$\mathrm{Kt}=0,26-0,27$

Nicht bestimmt 
achtet der vermehrten Bildung von Toxinen in den Geweben, das Blut rasch von diesen Stoffen befreit, indem sie mit dem Harn eliminirt werden.

Ich benutze die Gelegenheit, an dieser Stelle mitzutheilen, dass es uns gelang, analoge Erscheinungen, wo gleichzeitig mit der Störung der Oxydationsprocesse im Organismus bald ein Sinken, bald ein Steigen der Toxicität des Harns beobachtet wurde, experimentell an Thieren hervorzurufen. Ich behalte es mir vor, auf diese Erscheinungen bei der Veröffentlichung der experimentellen Untersuchungen näher einzugehen und zugleich das Wesentliche der Methode von Nencki und Sieber zur Untersuchung der Oxydationsprocesse zu besprechen; weiter will ich auch in Kürze die neuesten Anschauungen über die Processe der Metamorphose in den Zellen im Allgemeinen und über die Bedeutung der Oxydasen und Katalysatoren bei diesen Vorgängen anführen.

Jetzt sei es mir gestattet, darauf zurückzukommen, womit ich die vorliegende Abhandlung begonnen habe. Zur Zeit bilden die chemischbiologischen Untersuchungen unstreitig den interessantesten und fruchtbarsten Wey zur Erforschung der Processe, die im Organismus der Geisteskranken stattfinden. Leider kennen wir aber noch nicbt genügend solche Metlroden, mit Hilfe deren wir gerade in das Wesen der intimen Processe der Zellmetamorphose einzudringen vermögen. Die Methode von Nencki und Sieber, die Untersuchung der einzelnen Bestandtheile des Harns, und die Verhältnisse (Coefficienten) der verschiedenen Substanzen zu einander, das Studium der Toxicität des Harns und einige andere zur Anwendung kommende |Untersuchungsmethoden, gestatten uns jetzt, wenn auch nur theilweise, dem Verständniss über das Wesen der Processe bei geistigen Störungen näher zu kommen. Es unterliegt keinem Zweifel, dass die Psychiatrie nur auf diesem Wege von einer symptomatologischen Lntersuchung der Geisteskrankheiten zu einer natürlicheren, wissensehaftlichen Classification derselben gelangen kann, die auf der Kenntniss des Wesens derjenigen Veränderungen der lletamorphose im Organismus beruht, deren äussere Symptome die Geistesstörungen darstellen. Dann erst dürfte ein actives Eingreifen der Therapie dieser Leiden möglich werden. 